University of Nebraska - Lincoln

DigitalCommons@University of Nebraska - Lincoln

Nutrition \& Health Sciences Dissertations \&

Theses

Nutrition and Health Sciences, Department of

$5-2011$

\title{
A New Single Workbout Test to Estimate Critical Power and Anaerobic Work Capacity
}

Haley Bergstrom

University of Nebraska-Lincoln, hbergstrom@unlserve.unl.edu

Follow this and additional works at: https://digitalcommons.unl.edu/nutritiondiss

Part of the Dietetics and Clinical Nutrition Commons, and the Exercise Physiology Commons

Bergstrom, Haley, "A New Single Workbout Test to Estimate Critical Power and Anaerobic Work Capacity" (2011). Nutrition \& Health Sciences Dissertations \& Theses. 20.

https://digitalcommons.unl.edu/nutritiondiss/20

This Article is brought to you for free and open access by the Nutrition and Health Sciences, Department of at DigitalCommons@University of Nebraska - Lincoln. It has been accepted for inclusion in Nutrition \& Health Sciences Dissertations \& Theses by an authorized administrator of DigitalCommons@University of Nebraska - Lincoln. 


\title{
A NEW SINGLE WORKBOUT TEST TO ESTIMATE CRITICAL POWER AND ANAEROBIC WORK CAPACITY
}

\author{
by \\ Haley Bergstrom
}

\begin{abstract}
A THESIS
Presented to the Faculty of

The Graduate College at the University of Nebraska

In Partial Fulfillment of Requirements

For the Degree in Master of Science

Major: Nutrition and Health Sciences

Under the Supervision of Professor Terry J. Housh

Lincoln, Nebraska
\end{abstract}

May, 2011 
A NEW SINGLE WORKBOUT TEST TO ESTIMATE CRITICAL POWER AND

ANAEROBIC WORK CAPACITY

Haley C. Bergstrom, M.S.

University of Nebraska, 2011

Advisor: Terry J. Housh

The purpose of the present study was to develop a new 3-min, single workbout test to estimate critical power (CP) and anaerobic work capacity (AWC) using the Monark cycle ergometer with the resistance based on the individual's body weight. Twelve moderately-trained adults ( 6 men and 6 women; mean age $\pm \mathrm{SD}=23.2 \pm 3.5 \mathrm{yr}$ ) performed an incremental cycle ergometer test to exhaustion. The CP and AWC were estimated from the original work limit $\left(\mathrm{W}_{\text {lim }}\right)$ versus time limit $\left(\mathrm{T}_{\text {lim }}\right)$ relationship $\left(\mathrm{CP}_{\mathrm{PT}}\right)$ and a 3-min all-out test $\left(\mathrm{CP}_{3 \min }\right)$ against a fixed resistance and compared to $\mathrm{CP}$ and $\mathrm{AWC}$ estimated from the new 3-min tests on the Monark cycle ergometer $\left(\mathrm{CP}_{3.5 \%}\right.$ and $\left.\mathrm{CP}_{4.5 \%}\right)$. The resistance values for the $\mathrm{CP}_{3.5 \%}$ and $\mathrm{CP}_{4.5 \%}$ tests were set at $3.5 \%$ and $4.5 \%$ of the subject's body weight $(\mathrm{kg})$. The Mean differences between estimates of CP and AWC derived from the four methods $\left(\mathrm{CP}_{\mathrm{PT}}, \mathrm{CP}_{3 \min }, \mathrm{CP}_{3.5 \%}\right.$ and $\left.\mathrm{CP}_{4.5 \%}\right)$ were analyzed using separate one-way repeated measures ANOVAs with paired t-tests and Bonferroni corrections for post-hoc comparisons. The relationships among the four estimates of CP and AWC were described using Pearson product-moment correlations and separate zeroorder correlation matrices. The results indicated that there were no significant differences $(\mathrm{p}>0.05)$ among mean $\mathrm{CP}$ values for $\mathrm{CP}_{\mathrm{PT}}(178 \pm 47 \mathrm{~W}), \mathrm{CP}_{3.5 \%}(173 \pm 40 \mathrm{~W})$, and $\mathrm{CP}_{4.5 \%}(186 \pm 44 \mathrm{~W})$. The mean $\mathrm{CP}_{3 \min }(193 \pm 54 \mathrm{~W})$, however, was significantly greater 
than $\mathrm{CP}_{\mathrm{PT}}$ and $\mathrm{CP}_{3.5 \%}$. There were no significant differences in $\mathrm{AWC}$ for the $\mathrm{CP}_{\mathrm{PT}}(13412$ $\pm 6247 \mathrm{~J}), \mathrm{CP}_{3 \min }(10895 \pm 2923 \mathrm{~J})$, and $\mathrm{CP}_{4.5 \%}(9842 \pm 4394 \mathrm{~J})$. The AWC values for the $\mathrm{CP}_{\mathrm{PT}}$ and $\mathrm{CP}_{3 \min }$, however, were significantly greater than $\mathrm{CP}_{3.5 \%}(8357 \pm 2946 \mathrm{~J})$. The results of the present study indicated that $\mathrm{CP}$ and AWC could be estimated from a single 3-min workbout test on the Monark cycle ergometer with the resistance set at $4.5 \%$ of body weight. 


\section{ACKNOWLEDGEMENTS}

This manuscript would not have been possible without the guidance, direction, and support of my advisor, professors, classmates, friends and family.

I express my sincere gratitude to Dr. Terry Housh for his mentorship throughout this project and for encouraging my continued education. To Dr. Richard Schmidt for his encouragement and support, while challenging me to think critically. To Dr. Glen Johnson for his unyielding advice and clarity in the editing process. To Jorge Zuniga for the hours he patiently spent teaching me about software, equipment, and procedures, all the while, encouraging me to find my passion in research. To Clayton Camic for his insight and advice throughout the project. To Daniel Traylor for his enthusiasm for research and help with data collection. A very special thank you to my brother, Bjorn for encouraging me to pursue my dreams. I am eternally grateful to my parents, Mary and Jerry, for their unconditional love and support. They instilled in me the importance of faith, dedication, and hard work, while challenging me to be pursue my passion. I am so blessed to have this educational opportunity and I thank God for all the challenges, successes, and failures that have led me to this point. 



\section{TABLE OF CONTENTS}

LIST OF TABLES AND FIGURES ...........................................vii

CHAPTER I $\quad$ INTRODUCTION ...........................................

CHAPTER II $\quad$ REVIEW OF LITERATURE $\ldots \ldots \ldots \ldots \ldots \ldots \ldots \ldots \ldots \ldots \ldots . \ldots \ldots$

Development of critical power and anaerobic work capacity from the two-parameter model..................8

Metabolic factors associated with $\mathrm{CP}$ and AWC..........12

Factors effecting the estimation of $\mathrm{CP}$ and AWC........24

Development and validation of a 3-min all-out test

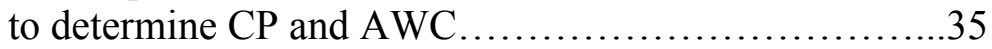

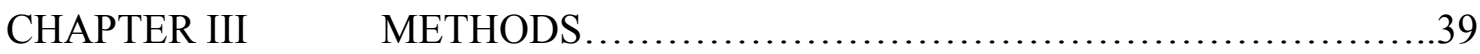

Subjects............................................

Experimental Approach and Design.......................39

Determination of $\dot{V} O_{2}$ peak and GET...................40

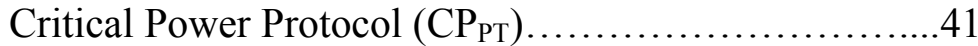

Three minute all-out test $\left(\mathrm{CP}_{3 \min }\right) \ldots \ldots \ldots \ldots \ldots \ldots \ldots \ldots . . .42$

Monark 3-min all-out tests $\left(\mathrm{CP}_{3.5 \%}\right.$ and $\left.\mathrm{CP}_{4.5 \%}\right) \ldots \ldots \ldots \ldots . .43$

Statistical Analysis....................................43

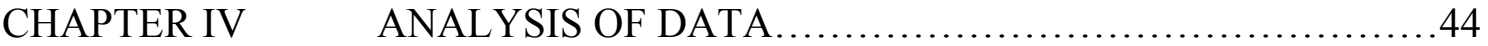

Results...........................................44

Discussion...............................................45

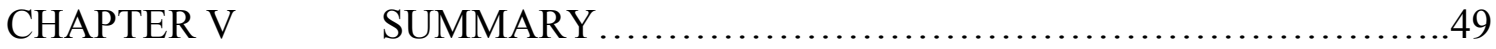

Statement of Purpose..........................................49

Procedures for Collection of Data..........................49

Analysis...................................................49

Findings..............................................50

Conclusions...........................................50

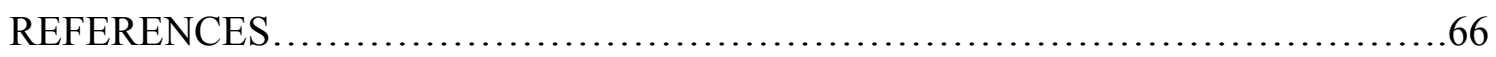

APPENDIX A

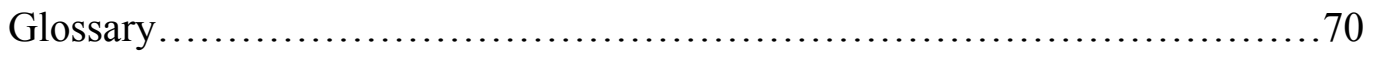

APPENDIX B

Statement of Informed Consent............................................ 71

Health History Questionnaire.............................................76 
LIST OF TABLES AND FIGURES

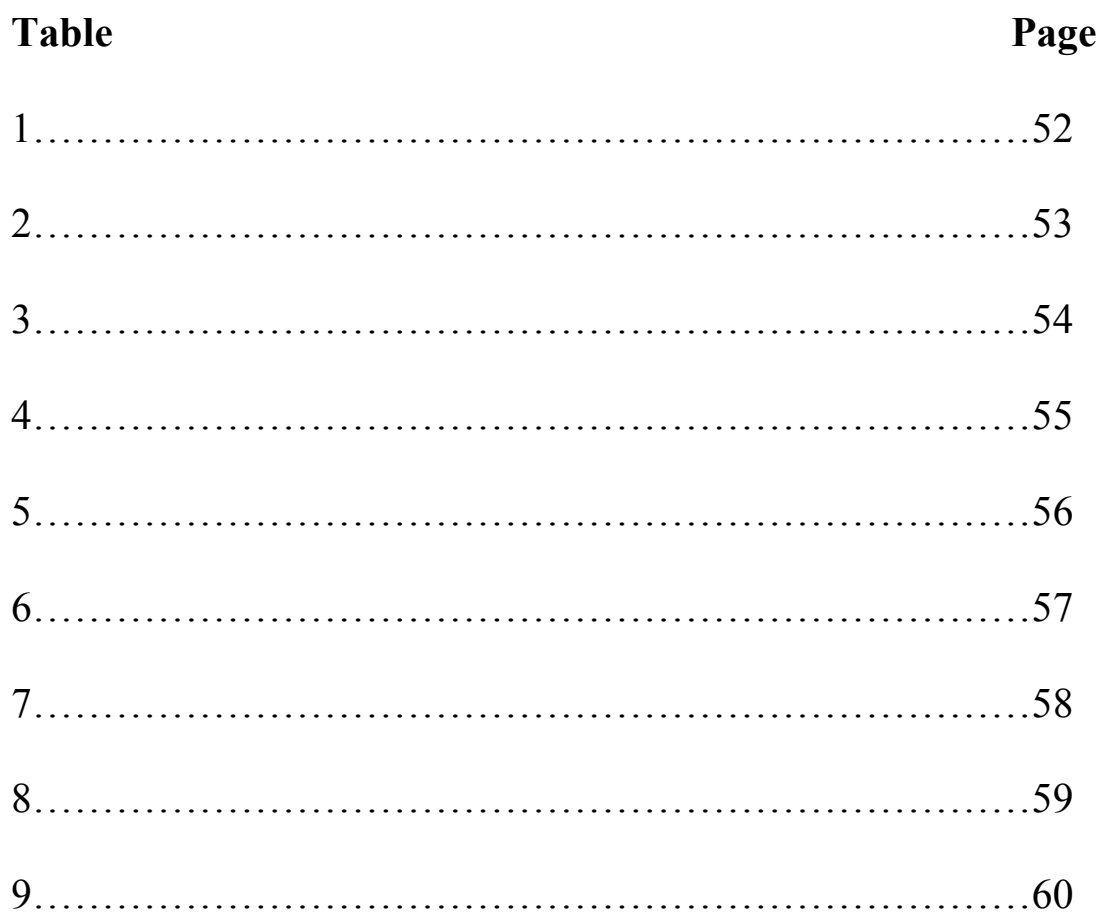

\section{Figure}

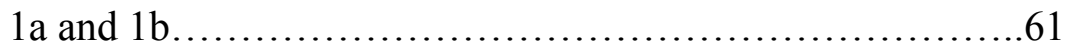

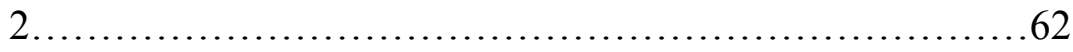

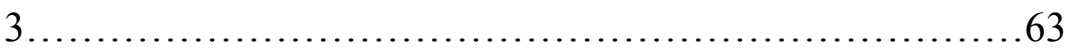

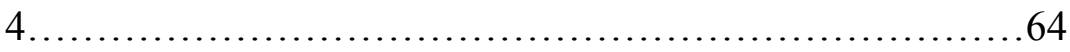

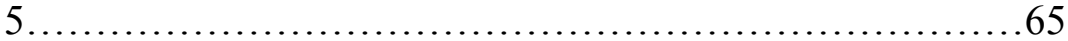




\section{CHAPTER I INTRODUCTION}

Monod and Scherrer (34) developed the critical power (CP) and critical force (CF) tests to quantify the amount of work a muscle or synergistic muscle group could perform before exhaustion during dynamic, isometric, and intermittent isometric muscle actions. For dynamic muscle actions, fatigue was quantified by the time to exhaustion or time limit $\left(\mathrm{T}_{\text {lim }}\right)$ at a given power output $(\mathrm{P})$. The critical power test relates the amount of work performed to exhaustion or work $\operatorname{limit}\left(\mathrm{W}_{\lim }=\mathrm{P} \times \mathrm{T}_{\lim }\right)$ and the $\mathrm{T}_{\lim }$ for a series of workbouts (Figure 1a). From the linear $\mathrm{W}_{\lim }$ versus $\mathrm{T}_{\text {lim }}$ relationship, two distinct parameters can be identified: the $\mathrm{CP}$ and anaerobic work capacity (AWC). The slope of the $\mathrm{W}_{\text {lim }}$ versus $\mathrm{T}_{\text {lim }}$ relationship defines the $\mathrm{CP}$ and the $\mathrm{y}$-intercept is the AWC. Theoretically, $\mathrm{CP}$ is the maximum $\mathrm{P}$ that can be maintained for an extended period of time without exhaustion and is equivalent to the asymptote of the hyperbolic $\mathrm{P}$ versus $\mathrm{T}_{\lim }$ relationship (Figure 1b). The AWC describes the total amount of work that can be accomplished utilizing only stored energy reserves within the muscle (34).

Moritani et al. (35) proposed a cycle ergometer analog of the $\mathrm{CP}$ test $\left(\mathrm{CP}_{\mathrm{PT}}\right.$ test) of Monod and Scherrer (34). During cycle crgometry, CP and AWC were determined from a series of rides to exhaustion at four different levels of $\mathrm{P}$. The $\mathrm{W}_{\text {lim }}$ was plotted versus $\mathrm{T}_{\text {lim }}$ to determine the slope (CP) and y-intercept (AWC). A highly linear relationship ( $\mathrm{r}>0.98$ ) was found for the $\mathrm{W}_{\lim }$ and $\mathrm{T}_{\text {lim }}$ relationship, which could be described by the equation: $\mathrm{W}_{\lim }=\mathrm{AWC}+\mathrm{CP}\left(\mathrm{T}_{\mathrm{lim}}\right)$ (Figure 1a) (35). Furthermore, there were significant correlations between the gas exchange threshold (GET) and $\mathrm{CP}(\mathrm{r}=$ $0.907-0.927)$ (34). Based on the correlation between GET and CP, the authors concluded that $\mathrm{CP}$ is dependent on oxygen supply (35). In addition, it has been suggested 
that the $\mathrm{AWC}$ is a measure of the total work that can be performed above $\mathrm{CP}$ and represents work produced from stored energy reserves within the muscle, such as ATP, phosocreatine, glycogen, and the oxygen bound to myoglobin $(8,34,35)$. It was determined that the $\mathrm{T}_{\text {lim }}$ at any $\mathrm{P}$ above $\mathrm{CP}$ could be estimated from the $\mathrm{CP}$ test parameters using the hyperbolic $\mathrm{P}$ versus $\mathrm{T}_{\text {lim }}$ curve (35). The $\mathrm{T}_{\mathrm{lim}}$ for any $\mathrm{P}$ was derived as follows:

$\mathrm{W}_{\text {lim }}=\mathrm{P}\left(\mathrm{T}_{\text {lim }}\right)$ and $\mathrm{W}_{\lim }=\mathrm{AWC}+\mathrm{CP}\left(\mathrm{T}_{\text {lim }}\right)$,

Thus, $\mathrm{P}\left(\mathrm{T}_{\mathrm{lim}}\right)=\mathrm{AWC}+\mathrm{CP}\left(\mathrm{T}_{\mathrm{lim}}\right)$ and $\mathrm{T}_{\lim }=\mathrm{AWC} /(\mathrm{P}-\mathrm{CP})($ Figure $1 \mathrm{~b})$.

The CP and AWC parameters identified by the CP test have a number of applications in laboratory and clinical settings. For example, $\mathrm{CP}$ has been used to demarcate the heavy from severe exercise intensity domains (37) and the CP and AWC parameters have been used to assess fitness for the prediction of exercise performance $(19,26,36)$.

A number of studies $(5,37,38,39)$ have provided evidence to suggest that $\mathrm{CP}$ demarcates the heavy from severe exercise intensity domains. For example, it has been suggested that $\mathrm{CP}$ represents the highest power output associated with the maintenance of intramuscular homeostasis $(29,37,39)$ including: 1$)$ the maintenance of muscle stores of phosphocreatine (PC), blood $\mathrm{pH}$ level, and bicarbonate; and 2) no significant increase in blood lactate, pulmonary $\dot{V} O_{2}$, and ventilation $(37,38,39)$. Furthermore, evidence has been found to indicate that the $\mathrm{CP}$ occurs at a similar intensity to the maximal lactate steady state (39). Therefore, these data suggest that $\mathrm{CP}$ represents the highest exercise intensity that can be maintained from only oxidative ATP production and demarcates the heavy from severe exercise intensity domains $(5,37,38,39)$. 
The $\mathrm{CP}$ test can be used to predict both anaerobic and aerobic exercise performance $(15,27,28,36,38,43,45)$. The AWC has been correlated with maximal oxygen deficit (19) and work performed in a $30 \mathrm{~s}$ all-out cycle ergometer test $(36,43)$. Thus, AWC provides a measure of anaerobic capabilities used to predict performance in power and sprint events (28). Alternatively, $\mathrm{CP}$ has been used to predict endurance capability $(15,27,38,45)$. Jenkins and Quigley $(27)$ found that training at or near CP for 8 weeks resulted in a 30\% increase in CP and a $7 \%$ increase in maximal oxygen consumption rate ( $\left.\dot{V} O_{2} \max \right)$. Thus, $\mathrm{CP}$ has been shown to be sensitive to endurance or interval training and increases in $\mathrm{CP}$ are associated with improved endurance performance $(15,27,38,46)$.

A number of studies $(22,24,25,33,37)$, however, have questioned the hypothesis that “... the critical power of a muscle (or muscle group) corresponds to the maximum rate it can keep up for a very long time without fatigue" (34, p. 329). For example, Housh et al. (22) found that $\mathrm{CP}$ was overestimated by $17 \%$ and could be maintained for a mean of only $33.31 \mathrm{~min} \pm 15.37 \mathrm{~min}$. In addition, Jenkins and Quigley (25) reported that only 2 of 8 subjects could maintain CP for 30 minutes. McLellen and Cheung (33) demonstrated that continuous cycle ergometery at the $\mathrm{CP}$ could be maintained for a mean time to exhaustion of $20.5 \mathrm{~min}$. Furthermore, Bull et al. (9) compared five different mathematical models to estimate $\mathrm{CP}$ and had the subjects ride to exhaustion at the lowest $\mathrm{CP}$ estimate from the models. The results indicated that 2 of 9 subjects did not complete 60 minutes of exercise at $\mathrm{CP}$ and the remaining subjects had a mean heart rate $92 \%$ of their maximal heart rate $(9)$. Other studies $(24,37)$ have found that $\mathrm{CP}$ overestimates the P associated with onset of blood lactate (OBLA) and the ventilatory anaerobic threshold. 
Housh et al. (24) found that CP was $28 \%$ higher than the P associated with the OBLA. In addition, $\mathrm{CP}$ was found to be $64 \%$ higher than the mean ventilatory anaerobic threshold (37). Thus, the results of multiple studies $(9,24,25,33,37)$ have indicated that exercise at $\mathrm{CP}$ is above the OBLA and ventilatory anaerobic threshold, and overestimates the maximal power output that can be maintained for an extended period of time without exhaustion.

A number of studies have modified the $\mathrm{CP}$ test protocol in an attempt to improve its validity. For example, various linear and nonlinear mathematical models have been used to identify the mathematical model that provides the lowest estimate of CP. Gaesser et al. (16) and Bull et al. (9) found that the three-parameter, nonlinear model ( $\mathrm{t}=\mathrm{AWC} / \mathrm{P}$ $\left.-\mathrm{CP})-\left(\mathrm{AWC} /\left(\mathrm{P}_{\max }-\mathrm{CP}\right)\right)\right)$ provided the lowest estimate of CP. Smith and Hill (41), however, found no differences in the estimates of CP from nonlinear and linear models. It has been suggested that differences between models in the estimation of CP $(9,16)$ may be the result of test protocols that utilize less than four prediction workbouts, multiple workbouts conducted on the same day, and/or workbouts that are all shorter than $10 \mathrm{~min}$ in duration (41). Hill et al. (19), however, indicated that the nonlinear and linear models provide similar estimates of $\mathrm{CP}$ when the procedure included two sets of five bouts of cycling exercise to exhaustion at varying $\mathrm{P}$, with each exercise test separated by $24 \mathrm{hrs}$. The results of these studies $(9,16,19,41)$ suggest the need to follow a common CP test procedure and that generally, the nonlinear, three-parameter model provides the lowest estimate of CP.

Other methodological considerations for the estimation of CP and AWC include the effects of cadence and manipulation of the power profile. Previous studies $(2,12,46)$ 
have indicated that $\mathrm{CP}$, estimated from the power duration relationship, and end test power (EP) were greater for lower than higher pedal cadence workbouts. There were, however, no significant differences between low and high cadence CP values estimated from the two-parameter linear model (2). In addition, Carnivale and Gaesser (12) found no significant difference in AWC estimates between low and high cadence workbouts. Vanhatalo et al. (46), however, reported that work done above end test power (WEP), an analog to AWC, was greater for lower pedal cadences. Furthermore, recent studies have reported that manipulation of the power profile had no effect on EP, WEP, or AWC $(18,46)$. Thus, the results of previous studies $(12,18,46)$ have indicated that CP, EP, and WEP were affected by variations in cadence, but not manipulation of the power profile.

The practical application of a multiple workbout $\mathrm{CP}$ test is limited by the physically demanding nature of the protocol and the need for subjects to perform $3-5$ exhaustive workbouts $(12,15,25,38,40,41)$. Therefore, manipulation of the number of predictive workbouts necessary to estimate $\mathrm{CP}$ and $\mathrm{AWC}$ has been proposed. For example, Housh et al. (23) found that as few as 2 exhaustive (1-10 min) workbouts could be used to accurately estimate $\mathrm{CP}$ and $\mathrm{AWC}$ if the times to exhaustion differed by approximately $5 \mathrm{~min}$. Recently, however, attempts have been made to develop a protocol that utilized a single maximal workbout to estimate $\mathrm{CP}$ and AWC. For example, Brickley et al. (6) attempted to identify CP from a single $90 \mathrm{~s}$ test. The authors, however, determined the test must be greater than $90 \mathrm{~s}$ to deplete anaerobic stores and force energy production from aerobic sources (6). Therefore, Burnley et al. (10) proposed a 3-min allout test $\left(\mathrm{CP}_{3 \min }\right.$ test) to identify EP that theoretically, represented the boundary between the heavy and severe exercise intensity domains. Rides to exhaustion were conducted at 
$15 \mathrm{~W}$ below and $15 \mathrm{~W}$ above EP (10). It was shown that subjects were able to maintain steady-state exercise at $15 \mathrm{~W}$ below EP, but exhausted during rides at $15 \mathrm{~W}$ above EP (10). As a result, Burnley et al. (10) suggested that a 3-min all-out test “...represents a promising method of identifying the maximal steady-state power output in a single test" (p. 2000).

Vanhatalo et al. (44) correlated the EP and WEP from the $\mathrm{CP}_{3 \min }$ test with $\mathrm{CP}$ and AWC, respectively, from the $\mathrm{CP}_{\mathrm{PT}}$ test. The results indicated that $\mathrm{EP}$ and $\mathrm{CP}$ were correlated at $r=0.99$, while WEP and AWC were correlated at $r=0.84$ (43). These data suggested that CP and AWC could be estimated from a 3-min all-out test $(10,44,45,46)$.

While a single 3-min all-out workbout is less physically demanding than the multiple exhaustive workbouts used to estimate $\mathrm{CP}$ and AWC from the linear $\mathrm{W}_{\text {lim }}$ versus $\mathrm{T}_{\text {lim }}$ model, the single workbout protocol of Vanhatalo et al. (44) requires a maximal cycle ergometer test to assess $\dot{V} O_{2}$ peak and gas exchange threshold (GET). These parameters are then used to determine the $\mathrm{P}$ for the $\mathrm{CP}_{3 \min }$ test to estimate $\mathrm{CP}$ and $\mathrm{AWC}$ $(44,45,46)$. A 3-min all-out test that utilizes a P based on body weight would eliminate the need for the measurement of gas exchange parameters and a maximal incremental cycle ergometer test and thus, provide a more practical way to estimate CP and AWC.

Therefore, the purpose of the present study was to develop a 3-min all-out test protocol using the Monark cycle ergometer for estimating CP and AWC with the resistance based on body weight. Based on previous studies $(10,34,35,44,45,46)$, we tested the hypothesis that a 3-min all-out test on a Monark Cycle ergometer, with the resistance set at $3.5 \%$ or $4.5 \%$ of the subjects body weight $\left(\mathrm{CP}_{3.5 \%}\right.$ and $\mathrm{CP}_{4.5 \%}$ tests), 
would provide estimates of $\mathrm{CP}$ and $\mathrm{AWC}$ that were not significantly different from the $\mathrm{CP}_{\mathrm{PT}}(35)$ and $\mathrm{CP}_{3 \min }$ tests $(10,44)$. 


\section{Chapter II}

\section{Review of Literature}

\section{Development of critical power and Anaerobic Work Capacity from the two- parameter model}

Monod and Scherrer (34)

The purpose of this article was to introduce the concept of critical power (CP) as it relates to the amount of static and dynamic work a synergic muscle group could perform without exhaustion. Critical power applied to synergic muscle groups has been defined as the maximum power output $(\mathrm{P})\left(\mathrm{W}_{\text {lim }}=\mathrm{P} \times \mathrm{T}_{\text {lim }}\right)$ that can be maintained without exhaustion. Limit work $\left(\mathrm{W}_{\mathrm{Lim}}\right)$ reflects the amount of work performed before exhaustion while limit time $\left(\mathrm{T}_{\mathrm{lim}}\right)$ defines the amount of time the work can be sustained. These parameters were identified by three separate constant power values performed to exhaustion. The power was held constant throughout the test. A mathematical model was used to linearly relate $\mathrm{W}_{\lim }$ and $\mathrm{T}_{\text {lim }}$ described by the equation $\mathrm{W}_{\lim }=a+b\left(\mathrm{~T}_{\text {lim }}\right)$. Three parameters were identified from this mathematical model: 1) The slope $(b)$ represents the critical power, or the amount of work that could be maintained for a very long time without exhaustion; 2) the y-intercept ( $a$ ) is the highest amount of work possible performed from the muscles energy reserves; 3) $\mathrm{T}_{\text {lim }}$ or time to exhaustion provides a prediction for any power output greater than $\mathrm{CP}$. 
Moritani et al. (35)

This study investigated whether the critical power (CP) concept could be applied to whole-body exercise as well as determine anaerobic threshold (AT) and $\dot{V} O_{2}$ max parameters. Eight male (20-33 years) and eight female (18-24 years) subjects were used in this study. An electrically-braked cycle ergometer was used to assess $\dot{V} O_{2}$ max and to identify the AT. Critical Power was determined from three workbouts performed at constant power outputs to exhaustion (determined to be when pedal cadence decreased to $\left.60 \mathrm{rev} \cdot \mathrm{min}^{-1}\right)$. The power outputs for males were 400, 350, 300, $275 \mathrm{~W}$, while 300, 250, 200 , and $175 \mathrm{~W}$ were used for females. The $\mathrm{W}_{\lim }\left(\mathrm{W}_{\lim }=\mathrm{P} \times \mathrm{T}_{\text {lim }}\right)$ was plotted against $\mathrm{T}_{\lim }$ and the regression equation $\mathrm{W}_{\lim }=\mathrm{a}+\mathrm{b}\left(\mathrm{T}_{\text {lim }}\right)$ was used to identify the slope $(\mathrm{CP})$ and $\mathrm{y}-$ intercept $(\mathrm{AWC})$. The relationship between $\mathrm{W}_{\text {lim }}$ and $\mathrm{T}_{\text {lim }}$ was highly linear $(\mathrm{r}>0.98)$. There were significant correlations observed between $\dot{V} O_{2} \max \left(\mathrm{ml} \cdot \mathrm{kg} \cdot \mathrm{min}^{-1}\right)$ and the CP (W) $(\mathrm{r}=0.919)$ and between $\dot{V} O_{2}$ at $\mathrm{CP}$ and $\dot{V} O_{2}$ at $\mathrm{AT}(\mathrm{r}=0.927)$. The mathematical model used to predict $\mathrm{CP}$ and $\mathrm{AWC}$ was used to predict $\mathrm{T}_{\lim }$ or the maximal time the exercise could be sustained. In this hyperbolic relationship, power output was plotted against $\mathrm{T}_{\text {lim. }}$. The $\mathrm{CP}$ was identified as the asymptote of the curve. The equation for the estimation of $\mathrm{T}_{\text {lim }}$ was derived as follows:

$\mathrm{W}_{\lim }=\mathrm{P}\left(\mathrm{T}_{\text {lim }}\right)$ and $\mathrm{W}_{\lim }=\mathrm{a}+\mathrm{b}\left(\mathrm{T}_{\mathrm{lim}}\right)$,

Thus, $\mathrm{P}\left(\mathrm{T}_{\mathrm{lim}}\right)=\mathrm{AWC}+\mathrm{CP}\left(\mathrm{T}_{\mathrm{lim}}\right)$ and $\mathrm{T}_{\mathrm{lim}}=\mathrm{AWC} /(\mathrm{P}-\mathrm{CP})$ 


\section{Nebelsick-Gullett et al. (36)}

The purpose of this study was to determine the reliability of the critical power (CP) test in the determination of anaerobic work capacity (AWC) compared to the Wingate anaerobic ergometer test (WAnT). In addition, this study sought to determine the test-retest reliability of the $\mathrm{CP}$ test. Twenty-five female subjects (Mean $\pm \mathrm{SD}$, age $=$ $21.88 \pm 2.49$ years) completed a WAnT on a Monarch bicycle ergometer for the determination of anaerobic capacity (AC). The WAnT consisted of an all-out $30 \mathrm{~s}$ effort with resistance set at $0.075 \mathrm{~kg} / \mathrm{kg}$ of body weight. The AC was expressed in watts (W) and defined as the total work performed during the 30 -s workbout ([resistance $(\mathrm{kg})$ x $6 \mathrm{x}$ number of revolutions x 2](6.12)). The subjects completed two CP tests. Each CP test consisted of 3 dynamic exercise bouts $(156-313 \mathrm{~W})$ against a fixed resistance at 80 rev $\cdot \mathrm{min}^{-1}$. The work limit (WL) versus time limit (TL) relationship (WL $\left.=\mathrm{P} \times \mathrm{TL}\right)$ was plotted and was shown to be highly linear $\left(r^{2}=0.98-1.00\right)$. Critical power was the power output corresponding to the slope of the WL-TL relationship and the y-intercept represented AWC. There was a significant relationship $(\mathrm{p}<0.05 ; \mathrm{r}=0.074)$ between AC and $\mathrm{AWC}$ and the test-retest correlations for $\mathrm{CP}(\mathrm{r}=0.94)$ and $\mathrm{AWC}(\mathrm{r}=0.87 ; \mathrm{p}<0.05)$ were significant. In addition, there were no significant differences between the test and retest means for $\mathrm{CP}$ or $\mathrm{AWC}($ Test $\mathrm{CP}=156 \pm 34 \mathrm{~W}$, Test $\mathrm{AWC}=9614 \pm 2735$ joules; Retest CP $=158 \pm 38 \mathrm{~W}$, Retest $\mathrm{AWC}=10083 \pm 2923$ joules $)$. The results of this study indicated that the Critical Power test is a valid measure of anaerobic work capacity. 
Housh et al. (22)

The purpose of this study was to examine the validity of the actual time to exhaustion $\left(\mathrm{AT}_{\text {lim }}\right)$ at various percentages of critical power $(\mathrm{CP})$ determined from the $\mathrm{CP}$ cycle ergometer test compared to the predicted time to exhaustion $\left(\mathrm{PT}_{\text {lim }}\right)$ derived from the power-curve analysis. Fourteen male subjects (Mean $\pm \mathrm{SD}$, age $=22.36 \pm 2.13$ years) volunteered to participate in this study. The $\mathrm{CP}$ was determined from four constant power output rides $\left(\right.$ range $=176-360 \mathrm{~W}$ ) at a pedal rate of $70 \mathrm{rev} \cdot \mathrm{min}^{-1}$ on a Monark cycle ergometer. The $\mathrm{W}_{\text {lim }}\left(\mathrm{P} \cdot \mathrm{T}_{\text {lim }}\right)$ was calculated for each of the CP rides. The CP test was used to calculate $\mathrm{PT}_{\text {lim }}$ from the formula $\mathrm{T}_{\text {lim }}=\mathrm{AWC} /(\mathrm{P}-\mathrm{CP})$. The subjects then completed rides at $\mathrm{CP}-20 \%, \mathrm{CP}, \mathrm{CP}+20 \%, \mathrm{CP}+40 \%$ and $\mathrm{CP}+60 \%$. The results of this study indicated that $\mathrm{AT}_{\text {lim }}$ and $\mathrm{PT}_{\text {lim }}$ were highly correlated $(\mathrm{r}=0.841$ to $\mathrm{r}=0.893$; $\mathrm{p}<0.05$ ) for the power loading above CP. The power loading that could be maintained for 60 min was estimated from the power curve (power output $=a x^{b}$ ) derived from the hyperbolic relationship between the four power loadings and $\mathrm{AT}_{\mathrm{lim}}$. Theoretically, $\mathrm{CP}$ represents a power loading capable of being maintained indefinitely without exhaustion. Therefore, the estimated power loading for $60 \mathrm{~min} \mathrm{PT}_{\text {lim }}$ was compared to the power loading $\mathrm{AT}_{\text {lim }}$ for the $\mathrm{CP}$. The mean $\mathrm{CP}$ of $197 \pm 39 \mathrm{~W}$ was significantly greater $(17 \%$; $\mathrm{p}<0.05)$ than the predicted value $(164 \pm 32 \mathrm{~W})$, while the mean $\mathrm{AT}_{\text {lim }}$ was $33.31 \pm 15.37$ min. The results of this study suggested the equation $\mathrm{T}_{\lim }=\mathrm{AWC} /(\mathrm{P}-\mathrm{CP})$ could be used to predict time to exhaustion for power loadings above CP. Furthermore, the power curve analysis indicated the $\mathrm{CP}$ derived from the $\mathrm{CP}$ test was $17 \%$ greater than the power loading that could be maintained for $60 \mathrm{~min}$. 


\section{Summary:}

The articles in this section indicate that a critical power (CP) and anaerobic work capacity (AWC) test can be applied to continuous muscle actions, intermittent muscle actions, and cycle ergometry $(34,35)$. These studies suggest a highly linear relationship exists between $\mathrm{W}_{\lim }$ and $\mathrm{T}_{\lim }(\mathrm{r}=0.98)$. This relationship is described by the equation $\mathrm{W}_{\text {lim }}=\mathrm{a}+\mathrm{b}\left(\mathrm{T}_{\text {lim }}\right)$. The slope (b) and the y-intercept (a) have been shown to represent $\mathrm{CP}$ and AWC, respectively (35). In general, $\mathrm{CP}$ has been defined as the maximum power output that can be maintained without exhaustion, while the AWC is described as the power output above $\mathrm{CP}$ that is derived from muscular energy reserves and independent of oxygen supply. The $\mathrm{T}_{\text {lim }}$ or time to exhaustion can be predicted from the hyperbolic relationship between the $\mathrm{CP}$ variables $\left(\mathrm{T}_{\mathrm{lim}}=\mathrm{AWC} /(\mathrm{P}-\mathrm{CP})\right.$. Thus, $\mathrm{CP}$ is the asymptote of the relationship between $\mathrm{T}_{\lim }$ and $\mathrm{P}(22,34,35,36)$.

\section{Metabolic Factors Associated with CP and AWC}

\subsection{Anaerobic Work Capacity}

$\underline{\text { Vandewalle et al. (43) }}$

The purpose of this study was to compare the amount of work performed $\left(\mathrm{W}_{\text {lim }}\right)$ versus the time to exhaustion $\left(\mathrm{T}_{\text {lim }}\right)$ relationship $\left(\mathrm{W}_{\lim }=\mathrm{a}+\mathrm{b}\left(\mathrm{T}_{\text {lim }}\right)\right.$. Nine men $($ Mean \pm $\mathrm{SD}$, weight $=71 \pm 5 \mathrm{~kg}$; age $26 \pm 4$ years) performed six exercise tests on a Monark bicycle ergometer. The subjects first completed a maximal aerobic power (MAP) test to exhaustion where resistance was increased by 5 Newtons $(\mathrm{N})$ every $3 \mathrm{~min}$ at $90 \mathrm{rev} \cdot \mathrm{min}^{-1}$. Power outputs derived from the MAP test were then used for the $\mathrm{W}_{\lim }-\mathrm{T}_{\text {lim }}$ test. This test involved four supramaximal tests to exhaustion $(105,120,135$, and $150 \%$ MAP) at 90 
$\mathrm{rev} \cdot \mathrm{min}^{-1}$. Anaerobic work (AW) was also measured from a single all-out $30 \mathrm{~s}$ anaerobic test at $1 \mathrm{~N} \cdot \mathrm{kg} \mathrm{BW}{ }^{-1}$. The $\mathrm{W}_{\mathrm{Lim}}-\mathrm{T}_{\mathrm{Lim}}$ produced a linear relationship $\left(\mathrm{W}_{\mathrm{Lim}}=\mathrm{a}+\mathrm{b} \cdot \mathrm{T}_{\mathrm{Lim}}\right)$. The average AW, however, was significantly larger than intercept $(a)(\mathrm{AW}=293 \mathrm{~J} \cdot \mathrm{kg}$ $\left.\mathrm{BW}^{-1}, a=205 \mathrm{~J} \cdot \mathrm{kg} \mathrm{BW}^{-1}, \mathrm{p}<0.05\right)$. Furthermore, the anaerobic capacity was not depleted in the $30 \mathrm{~s}$ all-out test as evidenced by a mechanical power twice MAP at the $30^{\text {th }}$ second. As a result of this study, the authors concluded the intercept $(a)$ of the $\mathrm{W}_{\text {lim- }}$ $\mathrm{T}_{\text {lim }}$ relationship did not provide an accurate estimation of anaerobic capacity. The authors suggested the underestimation of anaerobic power for the $\mathrm{W}_{\lim }-\mathrm{T}_{\text {lim }}$ relationship may be explained in part by the inertia of aerobic metabolism.

\section{Jenkins and Quigley (26)}

This investigation examined the relationship between the critical power $(\mathrm{CP})$ test and data collected from five one-min exercise bouts. Nine moderately active male subjects (Mean age $\pm \mathrm{SD}=18.8 \pm 1.2$ years) completed a CP test on a Monarch cycle ergometer. Three exercise bouts $(300,350$, and $400 \mathrm{~W})$ were then used to plot limits of work (kilojoules) versus time limit $(\mathrm{s})$ resulting in a highly linear relationship $(\mathrm{r}=0.99)$. AWC was then assessed via 5 one-min all-out cycle bouts against a fixed resistance of $0.075 \mathrm{~N} / \mathrm{kg}$ body mass. Total accumulated work was calculated (number of revolutions $\mathrm{x}$ applied resistance x 6) for the five work bouts. There was a significant correlation between the $y$-intercept of the work limit versus time limit relationship and the total work completed from the five maximal tests $(r=0.74 ; \mathrm{p}<0.05)$. The authors concluded that anaerobic work capacity was represented by the y-intercept derived from the work limittime limit relationship of the CP test. 


\section{Dekerle et al. (17)}

The purpose of this study was to compare the power-time relationship (W') to the total amount of work performed above critical power $\left(\mathrm{W}_{90 \mathrm{~s}}{ }^{\prime}\right)$ as well as the anaerobic work capacity, derived from a $90 \mathrm{~s}$ all-out test. Fourteen subjects $(10$ men and 4 women, mean $\pm \mathrm{SD}$, age $=30.5 \pm 1.7$ years; weight $=67.8 \pm 2.7 \mathrm{~kg}$ ) volunteered for this study. An incremental $\mathrm{ramp}\left(25 \mathrm{~W} \cdot \mathrm{min}^{-1}\right)$ protocol was used to assess $\dot{V} O_{2} \max ($ Mean $\pm \mathrm{SD}=$ $\left.3.9 \pm 0.7 \mathrm{~L} \cdot \mathrm{min}^{-1}\right)$ as well as the associated power output $\left(\mathrm{P}-\dot{V} O_{2} \max \right)$. Critical power and work (W') were calculated from three constant load tests to exhaustion $(103 \pm 3 \%, 97 \pm$ $3 \%$, and $90 \pm 2 \% \mathrm{P}-\dot{V} \mathrm{O}_{2} \max$ ) using the two-parameter, nonlinear model (power vs. time). The cadence was maintained at $90 \mathrm{rev} \cdot \mathrm{min}^{-1}$ and the test was terminated when cadence decreased below $85 \mathrm{rev} \cdot \mathrm{min}^{-1}$ for more than $5 \mathrm{~s}$. The subjects then performed a $90 \mathrm{~s}$ all-out test at the same self-selected cadence recorded from the ramp test $\left(93 \pm 3 \mathrm{rev} \cdot \mathrm{min}^{-1}\right)$. The $\mathrm{W}_{90}$ s was calculated as the power-time integral above $\mathrm{CP}$. The AWC was determined to be the difference between the actual power output of the 90 s all-out test and equivalent power output estimated from the max test. There was no significant difference between $\mathrm{W}^{\prime}$ and $\mathrm{W}_{90 \mathrm{~s}}$ ' $(\mathrm{P}=0.96)$. AWC, however, was significantly greater than both $\mathrm{W}^{\prime}$ and $\mathrm{W}_{90 \mathrm{~s}}{ }^{\prime}(\mathrm{P}=0.03$ and $\mathrm{P}=0.04$, respectively $)$. A significant correlation $(\mathrm{P}<0.001)$ was found between $\mathrm{W}^{\prime}$ and $\mathrm{AWC}, \mathrm{W}^{\prime}$ and $\mathrm{W}_{90 \mathrm{~s}}, \mathrm{~W}_{90 \mathrm{~s}}$ ' and $\mathrm{AWC}$. It was concluded that $\mathrm{W}^{\prime}$ and $\mathrm{W}_{90 \mathrm{~s}}$ ' are equivalent but underestimate $\mathrm{AWC}$. 


\subsection{Critical Power as a Measure of Endurance}

Jenkins and Quigley (25)

This investigation sought to validate critical power (CP) as a measure of a work rate that could be maintained for an appreciable amount of time without fatigue. A secondary objective was to determine whether $\mathrm{CP}$ corresponded to the maximal lactic acid threshold steady state. Eight highly trained male cyclists (Mean \pm SD age; $22 \pm 4 \mathrm{yr}$ ) completed an incremental cycle ergometer test to exhaustion for the determination of $\dot{V} O_{2}$ max. The subjects then completed four constant power output rides $(360,425,480$, and $520 \mathrm{~W}$ ) designed to elicit exhaustion within 15 minutes. Exhaustion was determined when the pedal rate decreased below $60 \mathrm{rev} \cdot \mathrm{min}^{-1}$ and could not be restored within $3 \mathrm{~s}$. Exercise duration (s) and total work performed (kilojoules, $\mathrm{kJ}$ ) were recorded and used to derive the equation for the linear relationship $(\mathrm{r} \geq 0.994)$ of total work $\left(\mathrm{W}_{\lim }\right)$ and time to exhaustion $\left(\mathrm{T}_{\mathrm{lim}}\right)$. The subjects then exercised for $30 \mathrm{~min}$ at $\mathrm{CP}($ mean $\pm \mathrm{SD} \mathrm{CP}=314.3 \pm$ $27.9 \mathrm{~W}$ ) and blood lactate was measured every $5 \mathrm{~min}$. If $\mathrm{CP}$ could not be sustained, the power output was reduced to allow the subjects to complete the exercise. Power output was also adjusted to maintain a blood lactate plateau. Blood lactate was measured from capillary blood taken from the left thumb at rest and after 5, 10,15, 20 and 30 min of exercise. Blood lactate rose steadily during the first 5-10 min of exercise and reached a plateau at $8.9 \pm 1.6 \mathrm{mmol} \cdot \mathrm{L}^{-1}$ for the last $20 \mathrm{~min}$ of exercise. Two subjects were able to complete 30 min of exercise at CP. For the other six subjects, power output was reduced $19.7 \pm 14.4 \mathrm{~W}(6.7 \%)$ below $\mathrm{CP}$. Mean lactate concentration $\left(8.9 \pm 1.6 \mathrm{mmol} \cdot \mathrm{L}^{-1}\right)$ and the $\mathrm{y}$-intercept were significantly correlated $(\mathrm{r}=0.78 ; \mathrm{P}<0.05)$. Theoretically, the $\mathrm{y}$ intercept of the work-limit versus time-limit relationship represents anaerobic capacity. 
The results of this study provided validation for a four-work bout model used to establish the $\mathrm{CP}$ curve. The actual mean $\mathrm{CP}$ for the 30 min test was overestimated (14.4W; 4.7\%) for the eight subjects. The authors concluded that $\mathrm{CP}$ provides a measure of exercise intensity that can be maintained continuously up to $30 \mathrm{~min}$ and that the $\mathrm{CP}$ test avoids methodological difficulties associated with lactate and ventilatory threshold testing and provides a high test-retest reliability $(\mathrm{r}=0.93)$.

\section{Housh et al. (24)}

The purpose of this study was to identify possible limitations to critical power (CP) from physiological mechanisms that include the onset of blood lactate (OBLA), the heart rate-workload slope (HR-WL), and the efficiency of electrical activity (EEA= slope of IEMG vs. workload). Twelve moderately to highly active males $(\mathrm{Mean} \pm \mathrm{SD}$, age $=$ $24.5 \pm 2.8$ years; height $=176.8 \pm 5.2 \mathrm{~cm}$; body weight $=79.5 \pm 10.6 \mathrm{~kg}$ ) completed two constant power output rides to exhaustion (392 and $314 \mathrm{~W}$ ) on a Monarch bicycle ergometer for the determination of CP. A discontinuous incremental bicycle ergometer test was then conducted at $70 \mathrm{rev} \cdot \mathrm{min}^{-1}$ with changes in work rate every two minutes (range 69 to $343 \mathrm{~W}$ ) in order to determine the OBLA, HR-WL slope and EEA. The OBLA was identified within 1 to 2 minutes upon completion of each work bout. There was a significant correlation between OBLA and $\mathrm{CP}(\mathrm{r}=0.616 ; \mathrm{p}<0.05)$. In addition, OBLA was determined to be a significant predictor of CP $(p<0.05)$. There were, however, significant differences in the power output at CP $(230.0 \pm 22.1 \mathrm{~W})$ and OBLA $(179 \pm 311.8 \mathrm{~W})$. Critical power was not predicted by the HR-WL slope $(0.342 \pm 0.071$ beats per watt) and EEA $(0.969 \pm 0.572$ microvolts per watt). Therefore, while the two 
threshold parameters, CP and OBLA, are significantly correlated and likely influenced by similar physiological factors, there are differences in the mechanisms behind these parameters.

\section{McLellan and Cheung (33)}

The individual anaerobic threshold (IAT) is the metabolic rate where elimination of blood lactate $(\mathrm{La})$ is equal to the rate of diffusion of La from the muscle to the blood. Theoretically, critical power (CP) and IAT occur at the same power output. Thus, the purpose of this study was to compare the metabolic response and the amount of work done at the IAT and CP. Fourteen males $($ Mean $\pm \mathrm{SD}$; age $=23.4 \pm 3.7 \mathrm{yr}$, weight $=75.0$ $\pm 10.6 \mathrm{~kg}$, height $=1.78 \pm 0.07 \mathrm{~m}$, and $\left.\dot{V} O_{2} \max =4.08 \pm 0.48 \mathrm{~L} \cdot \mathrm{min}^{-1}\right)$ completed at least 10 exercise tests on an electrically braked bicycle ergometer. An incremental ramp test $\left(30 \mathrm{~W} \cdot \mathrm{min}^{-1}\right)$ was performed to exhaustion for the determination of $\dot{V} O_{2}$ max and the IAT. Capillary blood samples from the earlobe in the last $30 \mathrm{~s}$ of each power output were used to determine blood La. The subjects then performed five constant power rides $(90,95$, 100,110 , and $120 \%$ of $\dot{V} O_{2} \max$ ) designed to elicit exhaustion within 2-15 minutes. Critical power was determined from the power output (W) versus time ${ }^{-1}$ relationship in which the intercept represented CP. Exercise tests at the power output defined by CP and IAT were then performed for $30 \mathrm{~min}$. In addition, nine of the subjects exercised at CP to examine the effects of a warm-up and hand position on CP. The calculation of CP had a test-retest reliability coefficient of 0.97 and there was a highly linear relationship $(0.95$ and 1.0) between power (W) and the inverse of time. There was a significant difference between the power output at IAT $(234.9 \pm 44.4 \mathrm{~W})$ and $\mathrm{CP}(265.1 \pm 39.3 \mathrm{~W}, \mathrm{p}<0.05)$. 
The time to exhaustion at the IAT $(29.9 \pm 0.47 \mathrm{~min})$ was significantly longer than time to exhaustion at CP $(20.5 \pm 4.5)$. In addition, blood lactate levels $\left(3.9 \pm 1.9 \mathrm{mmol} \cdot \mathrm{L}^{-1}\right) \mathrm{did}$ not change after 10 -min of exercise at IAT but increased during CP exercise $(5.0 \pm 1.1$ $\mathrm{mmol} \cdot \mathrm{L}^{-1}$ to $6.8 \pm 1.9 \mathrm{mmol} \cdot \mathrm{L}^{-1}$ ) from 5 to 15 minutes. The results of this study indicate that CP tests overestimate the power output associated with maximal steady-state blood lactate. Furthermore, CP is approximately $30 \mathrm{~W}$ greater than IAT and thus does not represent the same power output.

\section{Hill et al. (21)}

The purpose of this study was to 1$)$ compare critical power $\left(\mathrm{P}_{\text {critical }}\right)$ using the hyperbolic relationship between power and time to fatigue $\left(\mathrm{T}_{\text {fatigue }}\right)$, i.e., $\left.\mathrm{T}_{\text {fatigue }}=\mathrm{AWC} \cdot\left(\text { power }-\mathrm{P}_{\text {critical }}\right)^{-1}\right)$ to $\mathrm{P}^{\prime}{ }_{\text {critical }}($ the highest sustainable power output that does not elicit $\dot{V} O_{2}$ max determined from the hyperbolic relationship between power and time to $\dot{V} O_{2} \max \left(\mathrm{T} \dot{V} O_{2} \max \right)$, i.e., $\left.\mathrm{T} \dot{V} O_{2} \max =\mathrm{AWC} /\left(\mathrm{P}-\mathrm{P}^{\prime}{ }_{\text {critical }}\right)\right)$; and 2) determine if there was a linear relationship between $\mathrm{T}_{\text {fatigue }}$ and $\mathrm{T} \dot{V} \mathrm{O}_{2}$ max that could be used to identify the highest intensity for which tolerable exercise would elicit $\dot{V} O_{2}$ max. Eleven recreationally active subjects (Nine men; Mean $\pm \mathrm{SD}$; age $=25 \pm 6 \mathrm{yr}$, mass $=82.4 \pm 6.0$ $\mathrm{kg}$ and two women; age 18 and $21 \mathrm{yr}$, mass 57.2 and $59.9 \mathrm{~kg}$ ) performed exercise tests to exhaustion on an electrically-braked cycle ergometer. Power output, $\mathrm{T}_{\text {fatigue }}$, and $\dot{V} \mathrm{O}_{2} \max$ were recorded from four constant power tests $\left(110 \%, 135 \%, 95 \%\right.$, and $100 \%$ of $\left.\mathrm{P}_{\max }\right)$. Exercise tests were also conducted at $\mathrm{CP}$ and $\mathrm{CP}+10 \mathrm{~W}$. There was no significant difference and a high correlation $(\mathrm{P}=0.29 ; \mathrm{r}=0.93, \mathrm{p}<0.01)$ between $\mathrm{P}_{\text {critical }}$ and $\mathrm{P}^{\prime}$ critical 
$\left(\mathrm{CP}=198 \pm 44 \mathrm{~W}\right.$ and $\left.\mathrm{P}^{\prime}{ }_{\text {critical }}=196 \pm 42 \mathrm{~W}\right)$. The $\dot{V} O_{2}$ max was significantly lower at $\mathrm{P}_{\text {critical }}\left(2.90 \pm 0.57 \mathrm{~L} \cdot \mathrm{min}^{-1}\right)$ than the incremental test to exhaustion $\left(3.17 \pm 0.8 \mathrm{~L} \cdot \mathrm{min}^{-1}\right)$. The incremental $\dot{V} O_{2}$ max was not significantly different from exercise at $\mathrm{P}_{\text {critical }}+10 \mathrm{~W}$ $\left(3.03 \pm 0.60 \mathrm{~L} \cdot \mathrm{min}^{-1}\right)$. There was a strong relationship between $\mathrm{T}_{\text {fatigue }}$ and the $\mathrm{T} \dot{V} O_{2} \max$ $(\mathrm{r}=0.89 \pm 0.08)$. As a result, the authors concluded; 1$)$ the non-significant difference between $\mathrm{P}_{\text {critical }}$ and $\mathrm{P}_{\text {critical }}$ indicated that $\dot{V} O_{2}$ would increase to $\dot{V} O_{2}$ max at exercise above $\mathrm{P}_{\text {critical }}$ and $\mathrm{P}_{\text {critical: }}$ 2) both $\mathrm{P}_{\text {critical }}$ and $\mathrm{P}_{\text {critical }}$ demarcate the heavy-intensity from the severe-intensity domain; 3) the linear relationship between $\mathrm{T}_{\text {fatigue }}$ and $\mathrm{T} \dot{V} O_{2} \max$ could be used to predict the highest tolerable intensity capable of eliciting $\dot{\mathrm{V}} \mathrm{O}_{2}$ max.

\section{Pringle and Jones (39)}

This investigation sought to determine whether maximal lactate steady state (MLSS), critical power (CP), and electromyographic fatigue threshold (EMG $\mathrm{FT}_{\mathrm{FT}}$ ) occur at the same power output during cycle ergometry. In addition, metabolic and physiological responses (blood lactate [La], oxygen uptake, and integrated electromyogram (iEMG)) were measured at exercise above the power output at MLSS (P-MLSS). Eight regularly physically active subjects ( 7 male and 1 female, mean $\pm \mathrm{SD}$; age $=25 \pm 3$ years, mass $=$ $72.1 \pm 8.2 \mathrm{~kg}$ ) completed an incremental exercise test to exhaustion to determine lactate threshold $\left(\mathrm{Th}_{\mathrm{la}}\right)$ and $\dot{V} O_{2}$ max. MLSS was determined from four 30-min constant load workbouts (between $100 \%$ of the $\dot{V} O_{2}$ at the lactate threshold and $50 \%$ of the difference between the $\dot{V} O_{2}$ at the lactate threshold and $\dot{V} O_{2}$ max or $50 \% \Delta$ ) ( $\Delta$ is the magnitude of the interval between the $\dot{V} O_{2}$ at $\mathrm{Th}_{\mathrm{la}}$ and $\dot{V} O_{2}$ max). Critical power was estimated from 
four exhaustive (2-15 $\mathrm{min})$ constant load workbouts at power outputs (between 50\% $\Delta$ and $\left.110 \% \dot{\mathrm{V}} \mathrm{O}_{2} \mathrm{max}\right)$. The cadence was set at $90 \mathrm{rev} \cdot \mathrm{min}^{-1}$ and exhaustion was determined to be the point where cadence decreased below $85 \mathrm{rev} \cdot \mathrm{min}^{-1}$ for greater than $5 \mathrm{~s}$. There was a significant difference between the mean power output at CP and P-MLSS $(\mathrm{CP}=$ $242 \pm 25 \mathrm{~W}$ and P-MLSS $=222 \pm 23 \mathrm{~W} ; \mathrm{P}<0.05)$ but the two variables were highly correlated $(\mathrm{r}=0.95 ; \mathrm{P}<0.01)$. In addition, exercise at approximately $20 \mathrm{~W}$ above $\mathrm{P}$ MLSS caused a continued rise in [La], $\dot{V} O_{2}$ max, and minute ventilation. The authors concluded that, P-MLSS represented the upper limit of heavy exercise instead of CP. It is possible, however, that trained subjects inflated the $\mathrm{CP}$ measures because they were able to complete the workbouts to mechanical failure (inability to contract the exercising muscle) rather than voluntary exhaustion. Therefore, the differences in power outputs and physiological responses between CP and P-MLSS and the non-sustainability of exercise at $\mathrm{CP}$ was attributed to the training status of the subjects and the protocols for determination of MLSS and CP.

2.3 CP demarcates heavy-intensity from severe-intensity exercise domains Poole et al. (37)

The purpose of this study was to examine the respiratory and metabolic response to cycle ergometry for work performed at the power asymptote of the hyperbolic powerduration relationship $\left(\mathrm{P}_{\mathrm{LL}}\right)$ as well as work performed above this level. Eight untrained males $($ Mean $\pm \mathrm{SD}$; age $=22 \pm 1 \mathrm{yr}$; weight $=75.6 \pm 4.8 \mathrm{~kg})$ performed an incremental cycling test $\left(25 \mathrm{~W} \cdot \mathrm{min}^{-1}\right)$ to exhaustion for the estimation of lactic acid threshold $\left(\Theta_{\text {lac }}\right)$ and $\dot{V} O_{2}$ max. The $\mathrm{P}_{\mathrm{LL}}(197 \pm 12 \mathrm{~W})$ and the work performed above $\mathrm{P}_{\mathrm{LL}}\left(\mathrm{W}^{\prime}=14.6 \mathrm{~kJ}\right)$ 
were identified from a series of five square-wave tests to define the linear work-time relationship $\left(\mathrm{P}=\left(\mathrm{W}^{\prime} / \mathrm{t}\right)+\mathrm{P}_{\mathrm{LL}}\right)$. The subjects then performed a square-wave test at $\mathrm{P}_{\mathrm{LL}}$ and $\mathrm{P}_{\mathrm{LL}}+5 \%$. The $\mathrm{P}_{\mathrm{LL}}$ corresponded to $69 \%$ of the maximum power output $\left(\mathrm{P}_{\max }\right)$ achieved on the incremental test and was $164 \%$ of $\Theta_{\text {lac. }}$. Furthermore, the $\mathrm{P}_{\mathrm{LL}}$ was $46 \%$ of the difference between $\Theta_{\text {lac }}$ and $P_{\max }$. Constant load tests at $P_{L L}(197 \pm 12 W)$ were maintained for 24 minutes, while tests at $\mathrm{P}_{\mathrm{LL}}+5 \%$ averaged only $17.7 \pm 1.2 \mathrm{~min}$. A delayed steady state $\dot{V} O_{2}$ was reached with exercise at $\mathrm{P}_{\mathrm{LL}}$, however, at a power output greater than $\mathrm{P}_{\mathrm{LL}}, \dot{V} \mathrm{O}_{2}$ continued to rise and eventually reached $\dot{V} \mathrm{O}_{2}$ max. The results indicated that work between $\Theta_{\text {lac }}$ and $P_{L L}$ can be maintained for a prolonged exercise bout ( $>24$ min), while work done above $\mathrm{P}_{\mathrm{LL}}$ elicits fatigue within 24 minutes. Therefore, $\mathrm{P}_{\mathrm{LL}}$ demarcates the heavy-intensity (prolonged exercise despite increased acidosis) from the severe-intensity ( $\dot{V} \mathrm{O}_{2}$ max and the maximum level of metabolic acidosis).

\section{$\underline{\text { Hill and Smith (20) }}$}

The purposes of this study were: 1) to evaluate the relationship between power and time to reach $\dot{V} \mathrm{O}_{2}$ max during cycle ergometry; and 2) to compare CP (using the hyperbolic relationship between power and time to exhaustion, i.e., $\mathrm{T}_{\text {lim }}=\mathrm{AWC} /(\mathrm{P}-$ $\mathrm{CP})$ ) to $\mathrm{CP}^{\prime}$ (the highest power output without attainment of $\dot{V} \mathrm{O}_{2}$ max and derived from the hyperbolic relationship between power and time to $\dot{V} O_{2} \max$, i.e., $\mathrm{T} \dot{V} O_{2} \max =$ AWC'/(P-CP')). Eight subjects (6 women and 2 men, mean \pm SD: age, $24 \pm 2$ yrs; weight, $62.9 \pm 9.5$; height, $173 \pm 6 \mathrm{~cm})$ performed five exhaustive $(1-10 \mathrm{~min})$ constant power output rides (females: $3.5,4.5,5.5$, and $6.5 \mathrm{~W} \cdot \mathrm{kg}^{-1}$ and males: $4.0,5.5,7.0$, and 8.5 
$\mathrm{W} \cdot \mathrm{kg}^{-1}$ ) and a ride to exhaustion at $\mathrm{CP}$. Time to exhaustion, $\mathrm{CP}, \mathrm{CP}$, and $\dot{V} \mathrm{O}_{2}$ max were recorded for each constant power test. The CP $\left(170 \pm 44 \mathrm{~W}, 80 \% \dot{V} \mathrm{O}_{2} \max \right)$ and $\mathrm{CP}$ ' (167 $\pm 40 \mathrm{~W})$ were not statistically different $(\mathrm{p}<0.01)$ and highly correlated $(\mathrm{r}=0.95)$. Time to exhaustion at $\mathrm{CP}$ was $65.0 \pm 10.6 \mathrm{~min}$. The authors concluded that the non-significant difference between $\mathrm{CP}$ and $\mathrm{CP}$ ' supported $\mathrm{CP}$ as the highest sustainable power output that will not elicit $\dot{V} \mathrm{O}_{2}$ max. Therefore, $\mathrm{CP}$ demarcates the heavy-intensity from the severeintensity exercise domains.

\section{Brickley et al. (5)}

The purpose of this study was to examine the physiological response to exercise at critical power (CP) using blood lactate, oxygen uptake, and heart rate. In addition, the study tested the hypothesis that CP represents a work rate that can be maintained for at least 60 min. Seven trained males (Mean age \pm SD; $23.4 \pm 3.1$ years) completed five exercise tests on a Monarch bicycle ergometer. An incremental ramp test (25

$\left.\mathrm{W} \cdot \mathrm{min}^{-1}\right)$ to exhaustion was used to obtain $\dot{V} O_{2} \max \left(4.6 \pm 0.71 \cdot \mathrm{min}^{-1}\right)$ and the maximum power output $\left(\mathrm{P}_{\max }\right)(410 \pm 60 \mathrm{~W})$. Critical power was determined from three exhaustive (1-10-min) constant power output rides, on separate days, at work rates $(120 \%, 100 \%$, and $\left.95 \% \mathrm{P}_{\max }\right)$. The required cadence was determined from the preferred cadence chosen by the subjects during the incremental test (Mean \pm SD; $90 \pm 5 \mathrm{rpm}$ ). Fatigue was defined as the point at which cadence decreased $5 \mathrm{rev} \cdot \mathrm{min}^{-1}$ below the pre-determined cadence for 5 seconds. On a separate day an exercise test to exhaustion was conducted at each subject's CP and time to exhaustion was recorded to the nearest tenth. A highly linear relationship was observed $\left(\mathrm{R}^{2}=0.985\right)$ for the $\mathrm{CP}$ test $($ mean $\mathrm{CP}=273 \pm 38 \mathrm{~W})$. The 
time to exhaustion for the exercise test at CP ranged from $20 \mathrm{~min} 1 \mathrm{~s}$ to $40 \mathrm{~min} 37 \mathrm{~s}$ $($ Mean $\pm \mathrm{SD} ; 29 \min 34 \mathrm{~s} \pm 8 \mathrm{~min} 22 \mathrm{~s}$ ). At the end of the CP ride significant differences over time $(\mathrm{p}<0.001)$ were found in blood lactate concentration $(4.3 \pm 1.8$ to $6.5 \pm 2.0$ $\left.\mathrm{mmol} \cdot \mathrm{l}^{-1}\right)$, heart rate $\left(118 \pm 24\right.$ to $177 \pm 5$ beats $\left.\cdot \mathrm{min}^{-1}\right)$ and oxygen consumption $(3.7 \pm 0.6$ to $\left.4.1 \pm 0.51 \cdot \mathrm{min}^{-1}\right)$. The authors concluded that a steady-state was not achieved during exercise at CP. Therefore, they defined CP more accurately as the highest "non-steadystate" intensity that could be maintained between 20 and 40 minutes.

\section{Summary:}

The articles in this section described the anaerobic work capacity (AWC) derived from the y-intercept of the linear work-time relationship as a measure of the total work that can be performed above (CP) $(17,26,43)$. While AWC was correlated $(r=0.74)$ with the total work completed in one minute, it was significantly greater than $\mathrm{W}^{\prime}$ derived from the power-duration relationship and $\mathrm{W}_{90 \mathrm{~s}}$ ' derived from a $90 \mathrm{~s}$ all-out test (17).

Theoretically $\mathrm{W}^{\prime}$ and $\mathrm{W}_{90 \mathrm{~s}}$ ' described the work done above $\mathrm{CP}$, but underestimated $\mathrm{AWC}$ (17). Furthermore, critical power was examined as a measure of endurance. The predicted CP overestimated $(4.7 \%)$ the work rate that could be maintained for $>60 \min (25)$. Therefore, CP represents a power output that can be maintained continuously for approximately 20-40 min and will not elicit $\dot{V} O_{2} \max (25)$. In addition, CP was identified at a higher power output than both the OBLA and IAT (24). The CP, however, does demarcate the heavy-intensity from the severe-intensity domains $(5,28,37)$. 


\section{Factors Affecting the Estimation of $\mathrm{CP}$ and AWC}

3.1 Number of work bouts and length of predictive trials

Housh et al. (23)

The purpose of this investigation was to identify the number of work bouts necessary to accurately assess critical power (CP) and anaerobic work capacity (AWC). Twelve males (mean $\pm \mathrm{SD}=22.08 \pm 2.35$; range $=20$-27 years) performed four constant power-loading tests (172-360 W) to exhaustion on a Monark cycle ergometer. Two work bouts were performed per day followed by 24 hours of rest and the completion of the final two work bouts. The subjects pedaled at $70 \mathrm{rev} \cdot \mathrm{min}^{-1}$ against the fixed resistance and the test was terminated when the cadence fell to $65 \mathrm{rev} \cdot \mathrm{min}^{-1}$. The total time $\left(\mathrm{T}_{\text {lim }}\right)$ was recorded to the nearest $0.1 \mathrm{~s}$ and the total work $\left(\mathrm{W}_{\mathrm{LIM}}\right)$ was calculated as Power $(\mathrm{P})$ times $\left(\mathrm{T}_{\text {lim }}\right)$. CP was the slope (b) and AWC was the y-intercept (a) for the $\mathrm{W}_{\text {LIM }}-\mathrm{T}_{\mathrm{LIM}}$ relationship $\left(\mathrm{W}_{\lim }=\mathrm{a}+\mathrm{b}\left[\mathrm{T}_{\lim }\right]\right)$. All eleven possible combinations of two and three workbouts were used to determine $\mathrm{CP}$ and the values obtained were compared with the original CP $(199.12 \pm 37.16 \mathrm{~W})$ and AWC $(17,164.78 \pm 4,823.11 \mathrm{~J})$ determined from all four-power loadings. Only the combination of the second and third lowest power outputs produced a significant difference to the original four workbout $\mathrm{CP}(\mathrm{p}<0.05)$. CP derived from the highest and lowest power loadings was highly correlated $(\mathrm{r}=0.99$ for power loading $1,3,4$ and $r=0.98$ for power loading 1,4 ) with the value derived from the four power loadings. Therefore, the authors concluded that $\mathrm{CP}$ could be estimated in a single laboratory session from only two workbouts that elicited exhaustion within 1 to $10 \mathrm{~min}$ with the two workbouts differing by 5 min or more. 


\section{Bishop et al. (4)}

The purpose of this study was to determine if the length of selected predictive tests would elicit significant differences in the estimation of the critical power parameter derived from linear and hyperbolic models. Ten female subjects (mean $\pm \mathrm{SD}$; age $=18.6$ $\mathrm{yr}$, height $=171.1 \pm 0.03 \mathrm{~m}$, and weight $=66.8 \pm 6.2 \mathrm{~kg}$ ) performed five constant poweroutput exercise tests on an electronically-braked cycle ergometer. Power loadings were selected to elicit fatigue in approximately 1 to $10 \mathrm{~min}$. Critical power (CP) and work done above $\mathrm{CP}$ (W') were estimated from the linear $\mathrm{W}_{\lim }-\mathrm{t}_{\text {lim }}$ regression model as well as the $\mathrm{P}-\mathrm{t}_{\text {lim }}$ hyperbolic model. CP was calculated for both models in three ways: 1) using power outputs and time limit values from the first, third, and fifth $\mathrm{W}_{\lim }-\mathrm{t}_{\text {lim }}$ trials 2) values from the three highest power loadings, and 3) the values from the lowest three power loadings. There were significant differences $(\mathrm{P}<0.05)$ found for $\mathrm{CP}$ derived from the three different power output combinations for both the linear and hyperbolic models. $\mathrm{CP}$ derived from the highest three power output trials was greater than $\mathrm{CP}$ derived from the first, third, and fifth trials. The three lowest power-output trials produced the lowest $\mathrm{CP}$ values. Thus, the estimation of $\mathrm{CP}$ was highly dependent on the duration of the predictive trials. The authors suggested that aerobic inertia (the period in which aerobic metabolism is increasing to maximal levels) contributed to inflated values for $\mathrm{CP}$ from the three highest (shortest) trials. Therefore, the authors recommended that the predictive trials be at least greater than three min and calculated over a range of trial lengths to minimize the influence of aerobic inertia. 


\subsection{Effects of training of the CP parameters}

Jenkins and Quigley (27)

The purpose of the present study was to 1) examine critical power (CP) as a measure of endurance ability, 2) monitor the effects of 8 weeks of endurance training at $\mathrm{CP}$ on the slope (CP) and y-intercept (AWC), and 3) examine whether an increase in $\mathrm{CP}$ resulted in an increased ability to exercise at a higher intensity. Eighteen physically active untrained male cyclists were divided into two groups, an experimental group $(\mathrm{N}=12$, mean $\pm \mathrm{SD}$, age $=19.1 \pm 0.8 \mathrm{yr}$, height $=1.75 \pm 0.09 \mathrm{~m}$, weight $=72.9 \pm 6.4 \mathrm{~kg}$ ) and a control group $(\mathrm{N}=6$, age $=18.8 \pm 0.7 \mathrm{yr}$, height $=1.76 \pm 0.19 \mathrm{~m}$, weight $=70.7 \pm 4.6 \mathrm{~kg})$. An incremental cycle ergometer test to exhaustion was used to determine $\dot{V} O_{2}$ max. CP was determined from three constant work rate rides to exhaustion $(270,330$, and $390 \mathrm{~W})$. Each subject then completed a 40 min continuous cycle ergometery test at CP. If the subject could not maintain the power output at $\mathrm{CP}$, power was reduced (6-W increments) as necessary to allow for continuous cycling. The experimental group undertook an 8week training program $(30-40 \mathrm{~min}$ at $\mathrm{CP})$ and then retested $\dot{V} O_{2}$ max, $\mathrm{CP}$, and the 40 min ride. For the CP post-test, power output was increased for the experimental group's constant power output rides $(300,348,396 \mathrm{~W}$ or $330,372,414 \mathrm{~W})$, but remained the same for the control group $(270,330$, and $390 \mathrm{~W})$. As a result of endurance training, there was a $31 \%$ increase in $\mathrm{CP}(196 \pm 40.9 \mathrm{~W}$ to $255 \pm 28.4 \mathrm{~W})$ and an $8.5 \%$ increase in $\dot{V} O_{2} \max \left(49.2 \pm 7.8 \mathrm{ml} \cdot l^{-1} \cdot \mathrm{min}^{-1}\right.$ to $\left.53.4 \pm 6.4 \mathrm{ml} \cdot \cdot^{-1} \cdot \mathrm{min}^{-1}\right)$. Mean power output for the experimental group increased $28 \%$ from pre- to post-training for the 40 min endurance test $(190 \pm 34.5 \mathrm{~W}$ to $242 \pm 34.9, \mathrm{P}<0.001)$. The $40 \mathrm{~min} \mathrm{CP}$ test indicated $\mathrm{CP}$ was overestimated (6\%) for both the experimental and control group. There was a significant 
increase in the slope but not the $y$-intercept of the CP function $(\mathrm{P}<0.01)$. The results of this study indicate the CP function is sensitive to endurance training and that there is a strong relationship between endurance performance and $\mathrm{CP}$.

$\underline{\text { Vanhatalo et al. (45) }}$

This study examined the effect of 4 weeks of interval training on the critical power $(\mathrm{CP})$ profile derived from the standard $\mathrm{CP}$ test and a 3 min all-out test. Eight males and one female $($ Mean \pm SD: age $=29 \pm 6 \mathrm{yr}$, height $=1.77 \pm 0.08 \mathrm{~m}$, weight $=74.1 \pm$ $11.9 \mathrm{~kg}$ ) performed a ramp incremental test to exhaustion on an electronically-braked cycle ergometer for the determination of $\dot{V} \mathrm{O}_{2}$ peak and the gas exchange threshold (GET). The pretest protocol included a 3 min all-out test on an electronically-braked cycle ergometer with a resistance set at $50 \% \Delta$ (where $\Delta$ was the magnitude of the interval between GET and $\dot{V} \mathrm{O}_{2}$ peak, i.e., GET $+50 \% \Delta$ ) using the linear mode (linear factor $=$ $50 \% \Delta /$ cadence $^{2}$ ). End-test power (EP) and work done above (EP) were determined from this test. Three constant power output trials $\left(70 \% \Delta, 80 \% \Delta\right.$ and $100 \% \dot{V} O_{2}$ peak) were also used to predict $\mathrm{CP}$ and the curvature constant (W'). A training intervention was conducted three times a week for four weeks. Two of the weekly sessions included 5-min intervals at 105\% EP with 2.5 min active recovery and one weekly session of 10 repetitions of 2 min intervals $(P=50 \% \Delta \mathrm{WEP} / 120 \mathrm{~s}+\mathrm{EP})$. Post-training results were as follows: the 3 min all-out test showed a significant increase in EP (pretest $=225 \pm 52 \mathrm{~W}$ and post-test $=248 \pm 46 \mathrm{~W} ; \mathrm{p}<0.05)$ and no change in WEP $(\mathrm{P}=0.10)$ pre and post training. The CP significantly increased (pretest $=230 \pm 53 \mathrm{~W}$ and post-test $=255 \pm 50$ $\mathrm{W} ; \mathrm{p}<0.001)$. There was no change in $\mathrm{W}^{\prime}(\mathrm{p}<0.001)$. Furthermore, the CP and EP 
estimates before and after training were highly correlated (pre-training: $r=0.96$ and posttraining: $r=0.95 ; p<0.001)$. The increase in $\mathrm{CP}$ and $\mathrm{EP}$ were highly correlated but not significantly different $(r=0.77 ; p=0.57)$. The results of this study suggest $C P$ can be estimated from a 3-min all-out test and is sensitive to training-induced changes.

\subsection{Mathematical Models}

\section{Gaesser et al. (16)}

The purpose of this study was to compare estimates of critical power $\left(\mathrm{P}_{\mathrm{c}}\right)$ and anaerobic work capacity (W') parameters derived from five linear and non-linear models: 1) the two-parameter nonlinear, $\left.t=W^{\prime} /\left(P-P_{c}\right), 2\right)$ the three-parameter nonlinear model, $t$ $\left.\left.=\left(\mathrm{W}^{\prime} /\left(\mathrm{P}-\mathrm{P}_{\mathrm{c}}\right)\right)-\left(\mathrm{W}^{\prime} / \mathrm{P}_{\max }-\mathrm{P}_{\mathrm{c}}\right)\right), 3\right)$ the linear $\left.(\mathrm{P} \cdot \mathrm{t}), \mathrm{P} \cdot \mathrm{t}=\mathrm{W}^{\prime}+\left(\mathrm{P}_{\mathrm{c}} \cdot \mathrm{t}\right), 4\right)$ the linear $(\mathrm{P}), \mathrm{P}$ $=\left(\mathrm{W}^{\prime} / \mathrm{t}\right)+\mathrm{P}_{\mathrm{c}}$, and 5) the exponential model, $\mathrm{P}=\mathrm{P}_{\mathrm{c}}+\left(\mathrm{P}_{\max }-\mathrm{P}_{\mathrm{c}}\right) \exp (-\mathrm{t} / \mathrm{\Gamma})$. A secondary objective was to examine the correlation between $\mathrm{P}_{\mathrm{c}}$ and ventilatory threshold for longterm exercise $\left(\mathrm{LTE} \mathrm{T}_{\text {vent }}\right)$. Sixteen males $($ mean $\pm \mathrm{SD}$ : age $=21.1 \pm 1.3 \mathrm{yr}$, height $=180.1$ $\pm 8.2 \mathrm{~cm}$, and weight $=78.4 \pm 9.8 \mathrm{~kg}$ ) performed an incremental exercise test to exhaustion for the determination of peak power. The subjects then completed five to seven constant-load exercise bouts to exhaustion. Power-endurance time data were recorded and used to determine $\mathrm{P}_{\mathrm{c}}$ and $\mathrm{W}^{\prime}$ for the five mathematical models. Additionally, six subjects performed constant power exercise bouts in which ventilation and pulmonary gas exchange were monitored to identify the LTE $T_{\text {vent }}$. The $P_{c}$ and $W^{\prime}$ parameters derived from the different models were significantly different $(\mathrm{P}<0.005)$. The three-parameter nonlinear model produced the lowest $P_{c}(195 \pm 29 W)$. The exponential model provided the highest $\mathrm{P}_{\mathrm{c}}$ value $(242 \pm 21 \mathrm{~W})$. Alternatively, $\mathrm{W}^{\prime}$ was 
highest for the three-parameter nonlinear model $(58 \pm 19 \mathrm{~kJ})$ and lowest for the linear $(\mathrm{P})$ model $(18 \pm 5 \mathrm{~kJ})$. The $\mathrm{R}^{2}$ ranged from 0.96 for the linear $(\mathrm{P})$ model to 1.00 for the exponential model. Of the five mathematical models, the linear $(\mathrm{P})$ model was the only significantly different model. Correlations between $\mathrm{P}_{\mathrm{c}}$ for each model were high $(0.78$ to 0.91). The three-parameter model, however, did not correlate highly with $\mathrm{W}^{\prime}$ derived from the other four models. Critical power was significantly higher than the LTE $\mathrm{T}_{\mathrm{vent}}$ $(189 \pm 34 \mathrm{~W})$ for all but the three-parameter nonlinear model $(197 \pm 30 \mathrm{~W})$. There was a positive correlation for all five models $(r=0.69$ through 0.91$)$ between $P_{c}$ and LTE $T_{\text {vent }}$. The authors concluded that the three-parameter nonlinear model was the preferred model based on the assumption that: 1.) it was statistically strong, 2.) Power is not assumed to be infinite as time approaches 0 , and 3.) it provided a $P_{c}$ estimate that was close to the LTE $\mathrm{T}_{\text {vent, }}$ which indicates endurance capacity.

\section{Bull et al. (9)}

The purpose of this study was to compare critical power (CP) estimates from five different mathematical models and determine the time to exhaustion $(\mathrm{t})$ from the lowest $\mathrm{CP}$ estimate of the five models. Nine male subjects (mean $\pm \mathrm{SD}$ age $=25 \pm 3 \mathrm{yr}$, height $=$ $177 \pm 6 \mathrm{~cm}$, weight $=81.6 \pm 9.4 \mathrm{~kg}$ ) performed an incremental test to determine peak power (Ppeak) and peak heart rate (HRpeak) at exhaustion. $\mathrm{CP}$ was estimated from five trials with work rates chosen between Ppeak $-50 \mathrm{~W}$ and Ppeak $+50 \mathrm{~W}$ at a cadence of $60 \mathrm{rpm}$. Two linear $($ Linear-TW, TW $=\mathrm{AWC}+\mathrm{CP} \cdot \mathrm{t}$ and Linear-P, $\mathrm{P}=\mathrm{AWC} \cdot(1 / \mathrm{t})+\mathrm{CP})$, two non-linear (Non-linear-2, $\mathrm{t}=\mathrm{AWC} /(\mathrm{P}-\mathrm{CP})$ and Non-linear-3, $\mathrm{t}=\mathrm{AWC} /(\mathrm{P}-\mathrm{CP})$ $\left.\left(\mathrm{AWC} /\left(\mathrm{P}_{\max }-\mathrm{CP}\right)\right)\right)$, and one exponential mathematical model $\left(\mathrm{EXP}, \mathrm{P}=\mathrm{CP}+\left(\mathrm{P}_{\max }-\right.\right.$ 
$\mathrm{CP}) \exp (-\mathrm{t} / \tau)$ were used to predict $\mathrm{CP}$. The subjects then completed two trials to exhaustion, or $60 \mathrm{~min}$, at their lowest estimate of $\mathrm{CP}$ from the five models. The $\mathrm{CP}$ estimated from the Non-linear-3 model was significantly lower $(\mathrm{p}<0.05)$ than the other four models and was the lowest CP estimate for each subject. In addition, two subjects were not able to cycle at their $\mathrm{CP}$ for $60 \mathrm{~min}$. The mean time to exhaustion for the two subjects at CP-1 was 45.0 and $20.9 \mathrm{~min}$ and 48.1 and $18.0 \mathrm{~min}$ at $\mathrm{CP}-2$. The authors concluded that $\mathrm{CP}$ overestimates the power output that can be maintained for 60 minutes and thus does not represent a "fatigueless task."

\subsection{Effects of cadence and power Profile}

\section{Carnevale and Gaesser (12)}

The purpose of this study was to examine the effect of cadence $(60-100$ rev $\cdot \min ^{-1}$ ) on the power-duration relationship for cycle ergometery. Seven males (mean \pm SD; age $=20.4 \pm 0.3 \mathrm{yr}$, height $176.4 \pm 2.4 \mathrm{~cm}$, and $80.3 \pm 3.8 \mathrm{~kg}$ ) performed an incremental exercise test to exhaustion on a Monarch cycle ergometer to determine a range of power outputs to be used for the constant load critical power testing. Four work bouts were conducted at $60 \mathrm{rev} \cdot \mathrm{min}^{-1}$ at the following work rates: the highest work rate achieved in the incremental test, $30 \mathrm{~W}$ above and $30 \mathrm{~W}$ below the highest work rate, and $60 \mathrm{~W}$ below the highest work rate). Four exercise tests were then performed at 100 rev $\cdot \min ^{-1}$ as follows: the highest work rate achieved during the incremental test, $25 \mathrm{~W}$ above and below the highest rate, and $50 \mathrm{~W}$ above the highest rate. Critical power $\left(\theta_{\mathrm{PA}}\right)$ and anaerobic work capacity (W') were derived from the power-duration relationship $(\mathrm{t}=$ $\left.\mathrm{W}^{\prime} /\left(\mathrm{P}-\theta_{\mathrm{PA}}\right)\right)$. The results of this study indicated $\theta_{\mathrm{PA}}$ was significantly higher $(31 \mathrm{~W}$, 
$15.9 \%, \mathrm{P}<0.05))$ at $60 \mathrm{rev} \cdot \mathrm{min}^{-1}(235 \pm 8 \mathrm{~W})$ when compared to $100 \mathrm{rev} \cdot \mathrm{min}^{-1}(204 \pm 11$ W). There was no significant difference $(\mathrm{P}<0.05)$ between $\mathrm{W}^{\prime}$ performed at $60 \mathrm{rev} \cdot \mathrm{min}^{-}$ $(16.8 \pm 1.7 \mathrm{~kJ})$ and $100 \mathrm{rev} \cdot \min ^{-}(18.9 \pm 2.2 \mathrm{~kJ})$. Therefore, the authors concluded that for untrained subjects a low cadence $\left(60 \mathrm{rev} \cdot \mathrm{min}^{-1}\right)$ was superior to a high cadence $(100$ rev $\cdot \min ^{-1}$ ) for measures of sustainable power output during cycle ergometery. Furthermore, the authors indicated there was no effect of pedal cadence on the W' parameter.

Fukuba et al. (18)

The purpose of this study was to compare the work done above critical power (W') derived from the standard multiple constant power output tests with W' derived from two variable-power output cycle ergometer tests. Eleven male subjects (mean $\pm \mathrm{SD}$; age $=27 \pm 7 \mathrm{yr}$, height $=176 \pm 6 \mathrm{~cm}$, and weight $=68.7 \pm 4.8 \mathrm{~kg}$ ) performed a rampincremental test to exhaustion at $60 \mathrm{rev} \cdot \mathrm{min}^{-1}$ on an electrically-braked iso-power cycle ergometer. $\mathrm{CP}$ and $\mathrm{W}$ ' were estimated for each subject from four-to-six high-intensity square wave exercise bouts $\left(\mathrm{CP}=213 \pm 22.4 \mathrm{~W}\right.$ and $\left.\mathrm{W}^{\prime}=12.68 \pm 3.08 \mathrm{~W}\right)$. The subjects then performed a variable-power exercise bout termed the "UP" protocol in which they rode at $117 \%$ of $\mathrm{CP}$ for approximately half of $\mathrm{W}^{\prime}$ and then the work rate was increased to $134 \%$ of CP for the remainder of the test. Pedal cadence remained constant and exhaustion was indicated when cadence dropped to $50 \mathrm{rev} \cdot \mathrm{min}^{-1}$. A second variablepower protocol ("DOWN") was then performed. The subjects began the test at $134 \%$ of $\mathrm{CP}$ and power output was reduced to $117 \%$ of $\mathrm{CP}$ after exhaustion to approximately half of W'. The W' performed above CP for the "UP" protocol was $12.14 \pm 4.18 \mathrm{~kJ}$ and total 
W' for the "DOWN" protocol was $12.72 \pm 4.05 \mathrm{~kJ}$. There were no significant differences for the W' parameter derived from the three protocols (constant load P-t relationship, "UP”, or "Down" protocol) $(\mathrm{P}>0.05)$. The authors concluded that the non-significant differences for work done above CP between constant power output and varied-power output tests indicated that $\mathrm{W}^{\prime}$ is not affected by power variations in exhaustive cycle ergometery.

Vanhatalo et al. (46)

The purpose of this study was to investigate the effect of manipulations to the power profile and cadence on the end test power (EP) and work done above EP (WEP) parameters during a 3 min all-out cycle ergometer test. Nine subjects (8 males and one female, mean $\pm \mathrm{SD}$ : age $30 \pm 4$ years; weight $73.1 \pm 12.1 \mathrm{~kg}$; height $1.78 \pm 0.06 \mathrm{~m}$ ) performed an incremental ramp test to exhaustion for the assessment of $\dot{V} \mathrm{O}_{2}$ peak and the gas exchange threshold (GET). The subjects then performed one 'standard' 3-min all-out test in which the resistance was set using the linear factor of the ergometer ( $\mathrm{LF}=50 \% \Delta /$ preferred cadence $\left.{ }^{2}\right)$. The manipulation to cadence was tested $\left( \pm 20 \mathrm{rev} \cdot \mathrm{min}^{-1}\right)$ during two 3 min all-out tests against a fixed resistance at: 1$)$ a low cadence $(\mathrm{LF}=50 \% \Delta /$ (preferred cadence $\left.-10 \mathrm{rev} \cdot \mathrm{min}^{-1}\right)^{2}$ and 2$)$ a high cadence $(\mathrm{LF}=50 \% \Delta /($ preferred cadence +10 $\left.\mathrm{rev} \cdot \mathrm{min}^{-1}\right)^{2}$. Power profile manipulations included two 3-min tests where the power output was constant (100\% and $130 \%$ of the maximal power attained in the ramp test) for the initial $30 \mathrm{~s}$ followed by $2.5 \mathrm{~min}$ of all-out effort. The $\dot{V} \mathrm{O}_{2}$ peak was calculated during each test and defined as the highest $30 \mathrm{~s}$ average achieved during the test. The EP was calculated as the average power output of the final $30 \mathrm{~s}$ and WEP as the power-time 
integral over $180 \mathrm{~s}$ for each trial. The EP estimates $(100 \%=249 \pm 35 \mathrm{~W} ; 130 \%=245 \pm$ $39 \mathrm{~W} ; \mathrm{P}=0.32$ ) from the power profile manipulation trials were not significantly different from the standard 3-min all-out trial $(254 \pm 40 \mathrm{~W})$. Additionally, the manipulation of the power profile did not affect WEP $(\mathrm{P}=0.069)$. The high cadence trial (at an end-test cadence of $95 \pm 7 \mathrm{rev} \cdot \mathrm{min}^{-1}$ ), however, resulted in a significantly lower EP $(244 \pm 41 \mathrm{~W})$ compared to the standard end test cadence at $88 \pm 6 \mathrm{rpm}(254 \pm 40 \mathrm{~W})$. The low cadence trial $\left(77 \pm 5 \mathrm{rev} \cdot \mathrm{min}^{-1}\right)$ was not significantly different from the standard (251 $\pm 38 \mathrm{~W}$ ). The WEP for the low cadence trial was significantly higher while WEP for the high cadence trial was significantly lower $(p<0.05)$ than the standard test. The results of this study indicated that EP and WEP are robust to manipulations to the power profile and initial pacing. Manipulations to cadence, however, result in significant changes to the test parameters. Therefore, the $3 \mathrm{~min}$ all-out test is sensitive to variations in the ergometer resistance setting.

\section{Summary:}

The articles in this section described factors that may effect the estimation of critical power and anaerobic work capacity. The four constant load workbout CP protocol developed by Monod and Scherrer (1965) and refined by Moritani et al. (1981) has been manipulated in order to develop a less demanding procedure. Housh et al. (1990) determined CP from two exhaustive (1-10 min) workbouts. Furthermore, Bishop et al. (1998) proposed that the over prediction of CP may be reduced by trials lasting longer than 3 min and separated by at least five minutes. 
The effects of training on the CP parameters were determined from two training studies. An eight-week endurance training study indicated CP was sensitive to endurance training, while AWC was not (Jenkins and Quigley, 1992). Jenkins and Quigley (1992) also reported a $6 \%$ overestimation of $\mathrm{CP}$ from a 40 min ride to exhaustion. In a four-week high intensity interval training study, Vanhatalo et al. (2008a), found a significant increase in $\mathrm{CP}$ from pretest $(230 \pm 53 \mathrm{~W})$ to post-test $(255 \pm 50 \mathrm{~W}, \mathrm{p}<0.001)$. Vanhatalo et al. (2008a) reported a high correlation $(r=0.95)$ between $\mathrm{CP}$ and the end-test power (EP) determined from a three min all-out test, indicating a possible new method for the determination of CP.

The CP test parameters were determined and compared from five different mathematical models. Gaesser et al. (1995) identified the three-parameter nonlinear model, $\left.\mathrm{t}=\left(\mathrm{W}^{\prime} /\left(\mathrm{P}-\mathrm{P}_{\mathrm{c}}\right)\right)-\left(\mathrm{W}^{\prime} / \mathrm{P}_{\max }-\mathrm{P}_{\mathrm{c}}\right)\right)$, as the best predictor of CP. Bull et al. (2000), found the non-linear $\mathrm{t}=\mathrm{AWC} /(\mathrm{P}-\mathrm{CP})-\left(\mathrm{AWC} /\left(\mathrm{P}_{\max }-\mathrm{CP}\right)\right.$ underestimated $\mathrm{CP}$ and determined there to be a significant difference among all five models. In addition, Bull et al. (2000) determined CP overestimates the power output that can be maintained for 60 min and does not represent a "fatigueless task."

The effects of cadence and power profile manipulation were also considered. Carnevale and Gaesser (12) determined that CP parameters were better predicted at a low cadence $\left(60 \mathrm{rev} \cdot \mathrm{min}^{-1}\right)$ for untrained subjects and a high cadence $\left(100 \mathrm{rev} \cdot \mathrm{min}^{-1}\right)$ for trained subjects. A study by Fukuba et al. (18) indicated that manipulations to the power profile had no effect on the estimate of AWC. Furthermore, Vanhatalo et al. (46) found AWC was unaffected by power profile manipulation. The CP parameters, however, were altered by changes of the cadence. The culmination of these studies $(12,18,46)$ indicate 
that the $\mathrm{CP}$ parameters are sensitive to the duration or the trial, the number of trials, training, the mathematical model used, as well as power and cadence manipulations.

\section{Development and Validation of a 3-min all-out test to determine CP and AWC}

\section{Brickley et al. (6)}

The purpose of this investigation was to determine if maximal oxygen uptake ( $\left.\dot{V} \mathrm{O}_{2} \max \right)$ and submaximal aerobic ability (critical power) could be established from a 90-s all-out cycle ergometer test. Sixteen male subjects (Mean \pm SD, age: $30.4 \pm$ years; weight: $69.6 \pm 9.9 \mathrm{~kg}$ ) participated in this three-phase study. A ramp test to exhaustion on an electronically-braked cycle ergometer was completed to determine $\dot{V} O_{2}$ max. The subject then completed three constant power output tests to exhaustion for the estimation of critical power (CP). Power outputs were chosen to elicit exhaustion in 2-15 min. The pedal cadence was set at $90 \mathrm{rev} \cdot \mathrm{min}^{-1}$ and the test was terminated when cadence decreased below $85 \mathrm{rev} \cdot \mathrm{min}^{-1}$ for more than $5 \mathrm{sec}$. The $\mathrm{CP}$ was calculated from the linear model of work rate vs. 1/time. The $90 \mathrm{~s}$ all-out test required subjects to pedal at the selfselected cadence achieved during the ramp test $\left(94 \pm 3 \mathrm{rev} \cdot \mathrm{min}^{-1}\right)$. The subjects sprinted with the cadence imposed by the SRM system of the electronically-braked cycle ergometer. End power (EP) was determined from the final 10 s of the test (EP; $292 \pm 65$ W) and was significantly higher than $\mathrm{CP}(264 \pm 50 \mathrm{~W}$; $\mathrm{p}<0.01)$ determined from the $\mathrm{CP}$ test. Furthermore, the $\dot{V} O_{2}$ peak $\left(34335 \pm 682 \mathrm{~mL} \cdot \mathrm{min}^{-1}\right)$ at the end of the $90 \mathrm{~s}$ all-out test was significantly lower than the $\dot{V} O_{2} \max \left(3929 \pm 784 \mathrm{~mL} \cdot \mathrm{min}^{-1} ; \mathrm{p}<0.01\right)$ achieved during 
the ramp test. Therefore, it was concluded that a $90 \mathrm{~s}$ all-out test was of insufficient duration to elicit $\dot{V} O_{2}$ max or derive an EP output equal to $\mathrm{CP}$.

\section{Burnley et al. (10)}

The purpose of this study was to determine if 3 min of all-out exercise could provide a measure of the maximal steady-state power output to demarcate the heavyfrom severe-intensity domain and elicit a peak $\dot{V} O_{2}$ value consistent with measured oxygen consumption from a ramp test. Eleven recreationally active subjects (nine male, mean \pm SD: age $27 \pm 7 \mathrm{yr}$; height $1.76 \pm 0.10 \mathrm{~m}$; body mass $68.4 \pm 12.0 \mathrm{~kg}$ ) volunteered to participate in this study. The subjects visited the lab on six occasions with a minimum of 24 hours of recovery. A ramp protocol on an electronically-braked cycle ergometer was used to determine $\dot{V} O_{2}$ peak and the gas exchange threshold (GET). The subjects were then asked to perform a 3 min all-out test against a fixed resistance on an electronically-braked cycle ergometer. The resistance to pedaling was set using the linear factor of the ergometer $\left(\right.$ linear factor $=$ power $/$ cadence $\left.^{2}\right)$. The power output was $50 \% \Delta$ of the difference between GET and $\dot{V} O_{2}$ peak (GET $+50 \% \Delta$; where $\Delta$ is the magnitude of the interval between GET and $\dot{V} O_{2}$ peak). The cadence for the formula was the subjects preferred cadence $\left(80-90 \mathrm{rev} \cdot \mathrm{min}^{-1}\right)$ recorded during the ramp test. The three 3 min allout tests were used to establish the end-test power (EP) and work done above end power (WEP). The EP was the average power output over the last $30 \mathrm{~s}$ of the test. The subjects then performed two constant work rate tests up to $30 \mathrm{~min}$ at $15 \mathrm{~W}$ above and $15 \mathrm{~W}$ below end-test power (determined from the 3-min all-out test). Blood lactate was measured and steady state was defined as an increase of $<1.0 \mathrm{mM}$ in blood lactate from 10 to $30 \mathrm{~min}$ of 
exercise. The $\dot{V} \mathrm{O}_{2}$ peak was not statistically different between the ramp test (mean $\pm \mathrm{SD}=$ $\left.3.84 \pm 0.79 \mathrm{~L} \cdot \mathrm{min}^{-1}\right)$ and the $3 \mathrm{~min}$ all-out test $\left(3.78 \pm 0.68 \mathrm{~L} \cdot \mathrm{min}^{-1}\right)$. The average end-test power from the three $3 \mathrm{~min}$ all-out tests $(257 \pm 55 \mathrm{~W})$ was significantly lower than the power at the end of the ramp test $(368 \pm 73 \mathrm{~W})$. The EP was significantly higher than the power at the gas exchange threshold $(169 \pm 55 \mathrm{~W})$. Nine subjects were able to complete 30 min of exercise at the power output $15 \mathrm{~W}$ below EP while none completed the ride at $15 \mathrm{~W}$ above EP. It was concluded that a 3-min all-out cycle ergometer test elicited $\dot{V} \mathrm{O}_{2}$ peak. Furthermore, the data suggested a single test may be used as a measure of the maximal steady-state power output.

\section{Vanhatalo et al. (44)}

This investigation compared a 3-min all-out cycling test to the standard critical power test of four to five work bouts. The purpose of the study was to determine if the end test power (EP) and the work done above end test power (WEP) were equivalent to critical power (CP) and anaerobic work capacity (AWC), respectively. Ten subjects $($ mean $\pm \mathrm{SD}=$ age $33 \pm 9 \mathrm{yr}$, body mass $74.1 \pm 11.0 \mathrm{~kg}$, height $1.79 \pm 0.09 \mathrm{~m})$ accustomed to high-intensity exercise were used in this study. An incremental ramp protocol $\left(30 \mathrm{~W} \cdot \mathrm{min}^{-1}\right)$ was used to determine $\dot{V} O_{2}$ peak and the gas exchange threshold (GET). The $\dot{V} O_{2}$ peak was the highest $\dot{V} O_{2}$ during a 30 s period and GET was derived from $10 \mathrm{~s}$ averages using the V-slope method. The subjects performed two separate 3 min all-out tests against a fixed resistance to determine EP and WEP. The resistance was set at the power output of GET $+50 \% \Delta$ ( $\Delta$ was the magnitude of the interval between GET and $\dot{V} O_{2}$ peak). Five predicting trials were then performed at constant work rates to 
exhaustion to determine $\mathrm{CP}$ and the curvature constant parameter (W') derived from the work-time and 1/time linear models. There was no significant difference and a high correlation between $\mathrm{EP}$ of the 3 min all-out test (mean $\pm \mathrm{SD}=287 \pm 55 \mathrm{~W}$ ) and CP from the five work bouts $(287 \pm 56 \mathrm{~W} ; P=0.37, \mathrm{r}=0.99)$. Furthermore, the WEP calculated from the 3 -min all-out tests $(15.0 \pm 4.7 \mathrm{~kJ})$ was not significantly different from $\mathrm{W}^{\text {' }}$ derived from the five work bouts $(16.0 \pm 3.8 \mathrm{~kJ} ; P=0.35 ; \mathrm{r}=0.84)$. The results of this study indicate a 3 min all-out cycling test can provide an EP and WEP highly correlated with CP and W' respectively. Therefore, the authors suggested a 3 min all-out test may be used to derive the parameters of the power duration curve for a critical power test.

\section{Summary:}

The articles in this section focused on predicting critical power $(\mathrm{CP})$ and anaerobic work capacity (AWC) from a single work bout on a cycle ergometer. It was determined that the test must be greater than $90 \mathrm{~s}$ to deplete anaerobic stores and require energy production from aerobic sources (6). A 3 min all-out effort produced an end-test power (EP) that was not significantly different from, and highly correlated with CP derived from a standard test $(\mathrm{p}=0.37 ; \mathrm{r}=0.99)(44)$. Furthermore, the 3 min all-out test showed that work done above EP (WEP) was not significantly different from, and highly correlated with, AWC $(\mathrm{p}=0.35 ; \mathrm{r}=0.84)$. In addition, the 3 -min all-out test was further validated against the standard multiple work bout model (44). The results of this study (44) indicated that CP and EP were highly correlated $(r=0.96)$. Furthermore, an increase in CP from training was correlated with an increase in EP $(r=0.77)(44)$. In conclusion, the parameters of the original four-work bout model, CP and AWC, can be estimated from a 3 min all-out test. 


\section{CHAPTER III}

\section{METHODS}

\section{Subjects}

Twelve male and female subjects (mean age $\pm \mathrm{SD}=23.2 \pm 3.5 \mathrm{yr}$ ) were recruited for this study. The subjects were recreationally trained according to the ACSM Guidelines for Exercise Testing and Prescription (1), which include 20-30 minutes, 3-5 days per week, of moderate $\left(40-60 \% \dot{V} O_{2} \operatorname{Reserve}\left(\dot{V} O_{2} \mathrm{R}\right)\right)$ to vigorous $\left(>60 \% \dot{V} O_{2} \mathrm{R}\right)$ exercise. The study was approved by the University of Nebraska Institutional Review Board for Human Subjects. All subjects completed a health history questionnaire and signed a written informed consent document before testing.

\section{Experimental Approach and Design}

The subjects visited the laboratory on eight occasions, with $24-48 \mathrm{~h}$ between visits. During the first visit, the subjects performed an incremental cycle ergometer test to exhaustion for the determination of the $\dot{V} O_{2}$ peak and gas exchange threshold (GET). During the next four visits, the subjects completed the $\mathrm{CP}_{\mathrm{PT}}$ test, which included four randomly ordered, constant power output rides to exhaustion to determine critical power (CP) and anaerobic work capacity (AWC). The next visit included the 3-min, all-out test of Burnley et al. (10) $\left(\mathrm{CP}_{3 \min }\right.$ test) to determine end power output (EP) and work done above end power (WEP). Theoretically, the EP and WEP correspond to CP and AWC, respectively. The final two visits included 3-min, all-out tests on the Monark cycle ergometer, at randomly ordered resistances of $3.5 \%\left(\mathrm{CP}_{3.5 \%}\right.$ test $)$ and $4.5 \%\left(\mathrm{CP}_{4.5 \%}\right.$ test $)$ of body weight to estimate $\mathrm{CP}$ and AWC. 


\section{Determination of $\dot{V} O_{2}$ peak and GET (Visit 1)}

Each subject performed an incremental test to exhaustion on a calibrated Quinton (Corval 400) electronically-braked cycle ergometer (Quinton Instruments Inc., Seattle WA, USA) at a pedal cadence of $70 \mathrm{rev} \cdot \mathrm{min}^{-1}$. The ergometer seat height was adjusted so that the subject's legs were near full extension at the bottom of the pedal revolution. Toe clips were used to maintain pedal contact throughout the test. The same settings were used for subsequent tests on the electronically-braked cycle ergometer. All subjects wore a nose clip and breathed through a mouthpiece (2700; Hans Rudolph, Kansas City, MO, USA). Expired gas samples were collected and analyzed using a calibrated TrueMax 2400 metabolic cart (Parvo Medics, Sandy, UT, USA). The gas analyzers were calibrated with room air and gases of known concentration prior to all testing sessions. The $\mathrm{O}_{2}$, $\mathrm{CO}_{2}$, and ventilatory parameters were expressed as 10 -s averages. The subjects were fitted with a Polar Heart Watch system (Polar Electro Inc., Lake Success, NY). The test began at $30 \mathrm{~W}$ and the power output was increased by $30 \mathrm{~W}$ every 2 min until voluntary exhaustion or the subject's pedal rate decreased to below $70 \mathrm{rev} \cdot \mathrm{min}^{-1}$ for more than 10 seconds, despite strong verbal encouragement. The $\dot{V} \mathrm{O}_{2}$ peak was defined as the highest $\dot{V} O_{2}$ value in the last $30 \mathrm{~s}$ of the test that met two of the following three criteria (14): 1) $90 \%$ of age-predicted heart rate; 2 ) respiratory exchange ratio $>1.1$; and 3) a plateau of oxygen uptake (less than $150 \mathrm{~mL} \cdot \mathrm{min}^{-1}$ in $\dot{V} O_{2}$ over the last $30 \mathrm{~s}$ of the test).

The GET was determined using the V-slope method described by Beaver et al. (3). The GET was defined as the $\dot{V} O_{2}$ value corresponding to the intersection of two linear regression lines derived separately from the data points below and above the breakpoint 
in the $\dot{\mathrm{V} C \mathrm{O}_{2}}$ versus $\dot{\mathrm{V}} \mathrm{O}_{2}$ relationship (Figure 5). Power output values from the incremental test were plotted against $\dot{V} O_{2}$ values and the regression equation derived was used to determine the power outputs that correspond to the GET, 50,70 , and $80 \% \Delta$ (i.e., GET $+50 \% \Delta$, where $\Delta$ is the magnitude of the interval between GET and $\dot{V} o_{2}$ peak), as well as 100 and $105 \% \dot{V} O_{2}$ peak.

Critical Power Protocol (CP $\mathrm{CP}_{\mathrm{PT}}$ ) (Visit 2-5)

Critical power and AWC were determined on an electronically braked cycle ergomter, using the procedure of Moritani et al. (35) with the power outputs based on the recommendations of Vanhatalo et al. (44). The subjects rode to exhaustion at four separate power outputs equal to 70 and $80 \% \Delta$ (i.e., GET $+70 \% \Delta$ and $80 \% \Delta$, where $\Delta$ was the magnitude of the interval between GET and $\dot{V} o_{2}$ peak) and 100 and $105 \%$ $\dot{V} O_{2}$ peak (44). The subjects pedaled at $70 \mathrm{rev} \cdot \mathrm{min}^{-1}$ and the test was terminated when the subject could no longer maintain $65 \mathrm{rev} \cdot \mathrm{min}^{-1}$, despite strong verbal encouragement (23). The subjects were not aware of the power output or elapsed time during any of the rides. Linear regression using the two-parameter, work versus time model was used to determine $\mathrm{CP}$ and $\mathrm{AWC}$ from the four power outputs. Total work (or work limit; $\mathrm{W}_{\text {lim }}$ ) was calculated as the product of power output $(\mathrm{P})$ and time (or time limit; $\mathrm{T}_{\text {lim) }}$ ) to exhaustion (s). Figure 1a describes this model $\left(\mathrm{W}_{\lim }=\mathrm{a}+\mathrm{b}\left(\mathrm{T}_{\lim }\right)\right)$. The $\mathrm{CP}$ was defined as the slope (b) coefficient of the regression line (expressed in W), while AWC was the y-intercept (a) of this relationship (expressed in J). Theoretically, the CP is the asymptote of the hyperbolic $\mathrm{P}$ versus $\mathrm{T}_{\text {lim }}$ relationship (Figure $1 \mathrm{~b}$ ) defined by the equation $\mathrm{T}_{\lim }=$ $\mathrm{AWC} / \mathrm{P}-\mathrm{CP}$. This equation is derived as follows: 
$\mathrm{W}_{\lim }=\mathrm{P}\left(\mathrm{T}_{\lim }\right)$ and $\mathrm{W}_{\lim }=\mathrm{AWC}+\mathrm{CP}\left(\mathrm{T}_{\lim }\right)$

Thus, $\mathrm{P}\left(\mathrm{T}_{\text {lim }}\right)=\mathrm{AWC}+\mathrm{CP}\left(\mathrm{T}_{\text {lim }}\right)$ and

$\mathrm{T}_{\lim }=\mathrm{AWC} / \mathrm{P}-\mathrm{CP}$

Three-minute all-out test $\left(\mathrm{CP}_{3 \min }\right)$ (Visit 6)

Critical power and AWC from the $\mathrm{CP}_{3 \min }$ test were determined on the Calibrated Quinton Corval 400 (Quinton Instruments Inc., Seattle, WA) electronically braked cycle ergometer, using the procedures of Vanhatalo et al. (44). Critical power and AWC corresponded to EP and WEP, respectively (44). Each subject completed a warm-up at 50 $\mathrm{W}$ for 5 -min followed by $5 \mathrm{~min}$ of rest. The test began with unloaded cycling at 90 rev $\cdot \min ^{-1}$ for 3 min followed by a 3 min all-out effort. The subjects were instructed to increase the pedaling cadence to $110 \mathrm{rev} \cdot \mathrm{min}^{-1}$ in the last $5 \mathrm{~s}$ of the unloaded phase and then maintain the cadence as high as possible throughout the $\mathrm{CP}_{3 \min }$ test. The resistance for the $\mathrm{CP}_{3 \min }$ test was set using the linear mode of the Calibrated Quinton Corval 400 (Quinton Instruments Inc., Seattle, WA) electronically-braked cycle ergometer (linear factor $=$ power $/$ cadence $^{2}$ ). The linear factor was calculated as the power output halfway between $\dot{V} O_{2}$ peak and GET $(\mathrm{GET}+50 \% \Delta)$ divided by a cadence of $70 \mathrm{rev} \cdot \mathrm{min}^{-1}$ squared (10). Thus, the linear factor was equal to GET $+50 \% \Delta /\left(70 \mathrm{rev} \cdot \mathrm{min}^{-1}\right)^{2}$. To prevent pacing and ensure an all out effort, the subjects were not made aware of the elapsed time and strong verbal encouragement was provided.

The estimates of CP and AWC from the 3-min, all-out test were determined from the $\mathrm{P}$ versus time relationship (Figure 2). Critical power was the average $\mathrm{P}$ over the final $30 \mathrm{~s}$ of the test. The AWC was calculated as the integral of the P versus time relationship 
above CP using custom LabVIEW software (version 8.5, National Instruments, Austin, TX).

Monark 3-minute all-out tests $\left(\mathrm{CP}_{3.5 \%} \underline{\text { and } \mathrm{CP}_{4.5 \%}} \underline{\text { (Visit } 7)}\right.$

A Monark cycle ergometer (model 818 ) was used for the $\mathrm{CP}_{3.5 \%}$ and $\mathrm{CP}_{4.5 \%}$ test to estimate CP and AWC. The seat height was adjusted as previously described. The subject completed a warm-up at $1 \mathrm{~kg}$ for $5 \mathrm{~min}$. The test began when subject reached 110 rev $\cdot \min ^{-1}$ and the resistance was applied. The subjects pedaled with an all-out effort for 3 min. Resistances were randomized at 3.5\% $\left(\mathrm{CP}_{3.5 \%}\right)$ and $4.5 \%\left(\mathrm{CP}_{4.5 \%}\right)$ of body weight ( 0.035 or $0.045 \mathrm{x}$ body weight in $\mathrm{kg}$ ). The subject was not aware of the elapsed time or P. Strong verbal encouragement was provided. Pedal revolutions were recorded every $5 \mathrm{~s}$ using SMI software (Sports Medicine Industries, Inc., St. Cloud, MN, USA). The CP was the average power output for the final $30 \mathrm{~s}$ of the test and AWC was calculated as the integral of the $\mathrm{P}$ versus time relationship above CP (Figure 2).

$\underline{\text { Statistical analysis }}$

Mean differences between estimates of CP and AWC derived from the four methods $\left(\mathrm{CP}_{\mathrm{PT}}, \mathrm{CP}_{3 \min }, \mathrm{CP}_{3.5 \%}\right.$ and $\left.\mathrm{CP}_{4.5 \%}\right)$ were analyzed using separate one-way repeated measures ANOVAs with paired t-tests and Bonferroni corrections for post-hoc comparisons (Table 7). The relationships among the four estimates of $\mathrm{CP}$ and AWC were described using Pearson product-moment correlations and separate zero order correlation matrices (Table 8 and 9). The analyses were conducted using Statistical Package for the Social Sciences software (v.19.0. SPSS Inc., Chicago, Illinois, USA). 


\section{CHAPTER IV}

\section{ANALAYSIS OF DATA}

\section{$\underline{\text { Results }}$}

The descriptive characteristics of the subjects $(\mathrm{n}=12)$ are presented in Table 1.

The mean $( \pm \mathrm{SD}) \dot{V} O_{2}$ peak for the subjects in the present study was $3.02 \pm 0.73 \mathrm{~L} \cdot \mathrm{min}^{-1}$ $\left(42.97 \pm 7.42 \mathrm{~mL} \cdot \mathrm{kg}^{-1} \cdot \mathrm{min}^{-1}\right)$ (Table 2). The sample included six females $(2.66 \pm 0.63$ $\left.\mathrm{L} \cdot \mathrm{min}^{-1} ; 43.42 \pm 8.27 \mathrm{~mL} \cdot \mathrm{kg}^{-1} \cdot \mathrm{min}^{-1}\right)$ and six males $\left(3.38 \pm 0.67 \mathrm{~L} \cdot \mathrm{min}^{-1} ; 42.52 \pm 7.21\right.$ $\left.\mathrm{mL} \cdot \mathrm{kg}^{-1} \cdot \mathrm{min}^{-1}\right)$ (Tables 3 and 4). In addition, the mean $( \pm \mathrm{SD})$ maximal power output from the incremental test to exhaustion was $225 \pm 58 \mathrm{~W}$ (males, $257 \pm 51 \mathrm{~W}$; females, $210 \pm 42 \mathrm{~W}$ ) (Table 2) and the GET was $2.16 \pm 0.47 \mathrm{~L} \cdot \mathrm{min}^{-1}\left(30.73 \pm 4.02 \mathrm{~mL} \cdot \mathrm{kg}^{-1} \cdot \mathrm{min}^{-}\right.$ ${ }^{1}$ ). The GET occurred at $72 \pm 6 \%$ of $\dot{V} O_{2}$ peak (Table 5).

The mean $( \pm \mathrm{SD}) \mathrm{CP}_{\mathrm{PT}}$ was $178 \pm 47 \mathrm{~W}$ and the $\mathrm{r}^{2}$ values for the $\mathrm{W}_{\text {lim }}$ versus $\mathrm{T}_{\text {lim }}$ relationships ranged from $0.9804-0.9999$ (Table 6). There were no significant differences $(\mathrm{p}>0.05)$ among mean $\mathrm{CP}$ values for $\mathrm{CP}_{\mathrm{PT}}(178 \pm 47 \mathrm{~W}), \mathrm{CP}_{3.5 \%}(173 \pm 40$ $\mathrm{W})$, and $\mathrm{CP}_{4.5 \%}(186 \pm 44 \mathrm{~W})$ tests. The mean $\mathrm{CP}_{3 \min }$ test $(193 \pm 54 \mathrm{~W})$, however, was significantly greater than $\mathrm{CP}_{\mathrm{PT}}$ and $\mathrm{CP}_{3.5 \%}$ (Table 7). In addition, the $\mathrm{CP}$ values from the four tests were highly inter-correlated at $r=0.90-0.97$ (Table 8 ).

There were no significant differences in $\mathrm{AWC}$ for the $\mathrm{CP}_{\mathrm{PT}}(13412 \pm 6247 \mathrm{~J})$, $\mathrm{CP}_{3 \min }(10895 \pm 2923 \mathrm{~J})$, and $\mathrm{CP}_{4.5 \%}(9842 \pm 4394 \mathrm{~J})$. The $\mathrm{AWC}$ values for the $\mathrm{CP}_{\mathrm{PT}}$ and $\mathrm{CP}_{3 \min }$, however, were significantly greater than $\mathrm{CP}_{3.5 \%}(8357 \pm 2946 \mathrm{~J})$ (Table 7). The AWC values from the four protocols were highly inter-correlated at $0.76-0.91$ (Table 9). 


\section{Discussion}

The mean $( \pm \mathrm{SD}) \dot{V} O_{2}$ peak values for the male $\left(42.52 \pm 7.21 \mathrm{~mL} \cdot \mathrm{kg} \cdot \mathrm{min}^{-1}\right)$ and female $\left(43.42 \pm 8.27 \mathrm{~mL} \cdot \mathrm{kg}^{-1} \cdot \mathrm{min}^{-1}\right)$ subjects in the present study, resulted in cardiorespiratory fitness classifications of "fair" and "excellent," respectively (1). The mean GET for the total sample ( $\mathrm{n}=12$ ), which was used to set the power output for the $\mathrm{CP}_{3 \min }$ test, was $30.73 \pm 4.02 \mathrm{~mL} \cdot \mathrm{kg}^{-1} \cdot \mathrm{min}^{-1}$. These GET values $\left(72 \pm 6 \%\right.$ of $\dot{V} O_{2}$ peak) were typical of those previously reported for untrained to moderately trained (54-75\% $\mathrm{VO}_{2}$ peak) subjects $(11,13,42)$.

The $\mathrm{W}_{\text {lim }}$ and $\mathrm{T}_{\text {lim }}$ relationships (Figure 1a) for the $\mathrm{CP}_{\mathrm{PT}}$ tests were highly linear at $r^{2}=0.98-0.99$. These results were consistent with previous findings $(35,7,36,25)$ that have reported coefficients of determination of $r^{2}=0.982-1.000$ for the linearity of the $\mathrm{W}_{\text {lim }}$ and $\mathrm{T}_{\text {lim }}$ relationships. In addition, the range of $\mathrm{CP}_{\mathrm{PT}}$ values in the present study (103 to $265 \mathrm{~W}$ ) were similar to those of Moritani et al. (35) for untrained subjects (114 to 262 W), but less than those of more highly fit subjects (270 to $348 \mathrm{~W}$ ) (25). The range of AWC values from the $\mathrm{CP}_{\mathrm{PT}}$ test in the present study were 7,596 to $25,775 \mathrm{~J}$, which were similar to the values for untrained subjects $(6,777$ to $23,169 \mathrm{~J})$ reported in a previous study (Housh et al. 1989). These AWC values, however, were somewhat lower than those reported (10,300 to 30,500 J) by Jenkins and Quigley (20) for trained cyclists.

In the present study, the $\mathrm{CP}_{3 \min }, \mathrm{CP}_{3.5 \%}$, and $\mathrm{CP}_{4.5 \%}$ tests resulted in patterns of responses (Figures 2, 3, and 4) for the power output versus time relationships that were consistent with that of the 3 min, all-out test of Burnley et al. (10) and Vanhatalo et al. (44). The patterns for the power output versus time relationships for the $\mathrm{CP}_{3 \min }, \mathrm{CP}_{3.5 \%}$, and $\mathrm{CP}_{4.5 \%}$ involved initial increases in power output during the first 5 to 10 seconds, 
followed by steep declines throughout the first two minutes of the tests. The final one minute of the tests resulted in gradual decreases in power outputs that plateaued over the final 30 seconds.

We hypothesized that there would be no significant differences among CP estimates from the $\mathrm{CP}_{\mathrm{PT}}, \mathrm{CP}_{3 \min }, \mathrm{CP}_{3.5 \%}$, and $\mathrm{CP}_{4.5 \%}$ tests. The results showed no mean differences among the $\mathrm{CP}_{\mathrm{PT}}(178 \pm 47 \mathrm{~W}), \mathrm{CP}_{3.5 \%}(173 \pm 40 \mathrm{~W})$, and $\mathrm{CP}_{4.5 \%}(186 \pm 44$ W) tests. However, the mean $\mathrm{CP}$ value from the $\mathrm{CP}_{3 \min }$ test $(193 \pm 54 \mathrm{~W})$ was significantly $(\mathrm{p}=0.02)$ greater than the $\mathrm{CP}_{\mathrm{PT}}$ and $\mathrm{CP}_{3.5 \%}$ tests, but not significantly different from the $\mathrm{CP}_{4.5 \%}$ test (Table 7). These findings differed from the data of Vanhatalo et al. (44) who reported no significant difference between the $\mathrm{CP}_{3 \min }(287 \pm 55$ W) and the $\mathrm{CP}_{\mathrm{PT}}(287 \pm 56 \mathrm{~W})$ tests at a pedaling cadence of 80 to $90 \mathrm{rev} \cdot \mathrm{min}^{-1}$ for highly trained cyclists and runners. At a cadence of $100 \mathrm{rev} \cdot \mathrm{min}^{-1}$, however, Vanhatalo et al. (46) reported a lower $\mathrm{CP}$ value from the $\mathrm{CP}_{3 \min }$ test $(244 \pm 41 \mathrm{~W})$ than at 80 to 90 rev $\cdot \min ^{-1}(254 \pm 40 \mathrm{~W})$. It is possible, therefore, that the lower cadence used in the present study $\left(70 \mathrm{rev} \cdot \mathrm{min}^{-1}\right)$ contributed to the difference between the results of the present study and those of Vanhatalo (44). We selected a pedaling cadence of $70 \mathrm{rev} \cdot \mathrm{min}^{-1}$ because: 1 ) Previous studies $(31,32,30)$ have reported that untrained subjects typically select a preferred cadence of 60 to $80 \mathrm{rev} \cdot \mathrm{min}^{-1}$; and 2) Burnley et al. (10) set the resistance on the electronically-braked cycle ergometer using the same cadence as the incremental test to exhaustion. Future studies should examine the effect of pedaling cadence on differences between estimates of $\mathrm{CP}$ from the $\mathrm{CP}_{\mathrm{PT}}$ and $\mathrm{CP}_{3 \min }$ tests in trained and untrained subjects. 
The results of the present study indicated that there were no significant mean differences for estimates of $\mathrm{CP}$ and $\mathrm{AWC}$ from the $\mathrm{CP}_{\mathrm{PT}}, \mathrm{CP}_{3 \min }$, and $\mathrm{CP}_{4.5 \%}$ tests (Table 7). Furthermore, the $\mathrm{CP}$ values from these three tests were highly inter-correlated at $\mathrm{r}=$ 0.90-0.97 (Table 8). In addition, the AWC values were moderately to highly intercorrelated $r=0.76-0.91$ (Table 9). Thus, the current findings indicated that $\mathrm{CP}$ and AWC could be estimated from a 3-min, all-out test on a Monark cycle ergometer with the resistance set at $4.5 \%$ of the subject's body weight $\left(\mathrm{CP}_{4.5 \%}\right)$. A salient feature of the $\mathrm{CP}_{4.5 \%}$ test is that it requires only the measurement of the subject's body weight and a single workbout on a Monark cycle ergmometer. The orginal $\mathrm{CP}$ test requires a minimum of two, but usually three or more, rides to exhaustion on a cycle ergometer to estimate $\mathrm{CP}$ and AWC $(35,23)$. In addition, the method $\left(\mathrm{CP}_{3 \min }\right)$ proposed by Burnley et al. (10) requires the measurement and analysis of expired gas samples during an incremental test to exhaustion on an electronically-braked cycle ergometer, prior to the 3-min, all-out test. Therefore, the $\mathrm{CP}_{4.5 \%}$ test provides a more practical and easily administered method for determining $\mathrm{CP}$ and $\mathrm{AWC}$ than either the original procedure $\left(\mathrm{CP}_{\mathrm{PT}}\right)$ of Moritani et al. (35) or the single workbout alternative $\left(\mathrm{CP}_{3 \min }\right)$ of Burnley et al. (10).

The mean $\mathrm{CP}$ and $\mathrm{AWC}$ values estimated from the $\mathrm{CP}_{3.5 \%}$ test were significantly less than those of the $\mathrm{CP}_{3 \min }$ test (Table 7). Specifically, the $\mathrm{CP}_{3.5 \%}$ test underestimated $\mathrm{CP}$ and $\mathrm{AWC}$ by 10 to $23 \%$, respectively, compared to the $\mathrm{CP}_{3 \min }$ test. In addition, the $\mathrm{CP}_{3.5 \%}$ test underestimated AWC by $38 \%$ compared to the $\mathrm{CP}_{\mathrm{PT}}$ test. Even though there were no significant mean differences between the $\mathrm{CP}$ and $\mathrm{AWC}$ values from the $\mathrm{CP}_{3.5 \%}$ and $\mathrm{CP}_{4.5 \%}$ tests, the $\mathrm{CP}_{4.5 \%}$ test is recommended because of its similarities to the results of the $\mathrm{CP}_{\mathrm{PT}}$ and $\mathrm{CP}_{3 \min }$ tests. Thus, the current findings indicated that using a resistance of 
$4.5 \%$ of body weight provided more accurate estimates of CP and AWC than using a resistance of $3.5 \%$ of body weight when compared to the original CP test of Moritani et al. (35) and the single, workbout, 3-min, all-out CP test of Burnley et al. (10).

In summary, the results of the present study indicated that CP and AWC could be estimated from a single, 3-min, all-out, test on a Monark cycle ergomter with the resistance set at $4.5 \%$ of the subject's body weight. In the present study, it was found that: 1) Unlike Vanhatalo et al. (44), there was a significant mean difference between estimates of $\mathrm{CP}$ from the $\mathrm{CP}_{\mathrm{PT}}$ and $\mathrm{CP}_{3 \min }$ tests, possibly due to the lower pedaling cadence selected in this study; and 2) CP and AWC could be accurately estimated from the $\mathrm{CP}_{4.5 \%}$ test, but not the $\mathrm{CP}_{3.5 \%}$ test. Thus, the advantages of the $\mathrm{CP}_{4.5 \%}$ test over the $\mathrm{CP}_{\mathrm{PT}}$ and $\mathrm{CP}_{3 \min }$ tests are: 1$)$ The $\mathrm{CP}_{4.5 \%}$ tests requires only one workbout compared to multiple, exhaustive workbouts for the original $\mathrm{CP}$ test $\left(\mathrm{CP}_{\mathrm{PT}}\right)$ of Moritani et al. (35); 2) The $\mathrm{CP}_{4.5 \%}$ test utilizes a resistance set according to the body weight of the subject as opposed to the need to collect and analyze gas exchange variables during an incremental test to exhaustion to set the resistance for the $\mathrm{CP}_{3 \min }$ test of Burnley et al (10); and 3) The $\mathrm{CP}_{4.5 \%}$ test involves a single, 3-min, all-out, workbout on a Monark cycle ergometer, while the $\mathrm{CP}_{3 \min }$ test requires an electronically-braked cycle ergometer. Therefore, the $\mathrm{CP}_{45 \%}$ test is recommended as a new practical and accurate method for estimating $\mathrm{CP}$ and AWC. 


\section{CHAPTER V}

\section{SUMMARY}

\section{Statement of Purpose}

The purpose of the present study was to develop a new 3-min, single workbout test to estimate critical power (CP) and anaerobic work capacity (AWC) using the Monark cycle ergometer with the resistance based on the individual's body weight.

\section{Procedures for Collection of Data}

Twelve moderately-trained adults ( 6 men and 6 women; mean age $\pm \mathrm{SD}=23.2 \pm$ $3.5 \mathrm{yr}$ ) volunteered for to perform an incremental cycle ergometer test to exhaustion for the collection and analysis of gas exchange variables. Critical power and AWC were estimated from four separate procedures.

\section{$\underline{\text { Analysis }}$}

Mean differences between estimates of CP and AWC derived from the four methods $\left(\mathrm{CP}_{\mathrm{PT}}, \mathrm{CP}_{3 \min }, \mathrm{CP}_{3.5 \%}\right.$ and $\left.\mathrm{CP}_{4.5 \%}\right)$ were analyzed using separate one-way repeated measures ANOVAs with Bonferroni post-hoc comparisons. The relationships among the four estimates of $\mathrm{CP}$ and $\mathrm{AWC}$ were described using Pearson product-moment correlations and separate zero order correlation matrices. 
$\underline{\text { Findings }}$

$\dot{V} \mathrm{O}_{2}$ max, maximal power output, and GET

The mean $( \pm \mathrm{SD}) \dot{V} O_{2} \max$ for the subjects in the present study was $3.02 \pm 0.73$

$\mathrm{L} \cdot \mathrm{min}^{-1}\left(42.97 \pm 7.42 \mathrm{~mL} \cdot \mathrm{kg} \cdot \mathrm{min}^{-1}\right)$. The mean $( \pm \mathrm{SD})$ maximal power output from the incremental test to exhaustion was $225 \pm 58 \mathrm{~W}$ (males, $257 \pm 51 \mathrm{~W}$; females, $210 \pm 42$ W) (Table 2) and the GET was $2.16 \pm 0.47 \mathrm{~L} \cdot \mathrm{min}^{-1}\left(30.73 \pm 4.02 \mathrm{~mL} \cdot \mathrm{kg} \cdot \mathrm{min}^{-1}\right)$. The GET occurred at $72 \pm 6 \%$ of $\dot{V} O_{2} \max$.

\section{Critical Power}

The mean $( \pm \mathrm{SD}) \mathrm{CP}_{\mathrm{PT}}$ was $178 \pm 47 \mathrm{~W}$ and the $\mathrm{r}^{2}$ values for the $\mathrm{W}_{\text {lim }}$ versus $\mathrm{T}_{\text {lim }}$ relationships ranged from $0.9804-0.9999$. There were no significant differences $(\mathrm{p}>$ 0.05) among mean $\mathrm{CP}$ values for $\mathrm{CP}_{\mathrm{PT}}(178 \pm 47 \mathrm{~W}), \mathrm{CP}_{3.5 \%}(173 \pm 40 \mathrm{~W})$, and $\mathrm{CP}_{4.5 \%}$ $(186 \pm 44 \mathrm{~W})$. The mean $\mathrm{CP}_{3 \min }(193 \pm 54 \mathrm{~W})$, however, was significantly greater than $\mathrm{CP}_{\mathrm{PT}}$ and $\mathrm{CP}_{3.5 \%}$. In addition, the $\mathrm{CP}$ values from the four protocols were highly intercorrelated at $0.90-0.97$. Anaerobic Work Capacity

There were no significant differences in AWC for the $\mathrm{CP}_{\mathrm{PT}}(13,412 \pm 6,247 \mathrm{~J})$, $\mathrm{CP}_{3 \min }(10,895 \pm 2,923 \mathrm{~J})$, and $\mathrm{CP}_{4.5 \%}(9842 \pm 4394 \mathrm{~J})$. The $\mathrm{AWC}$ values for the $\mathrm{CP}_{\mathrm{PT}}$ and $\mathrm{CP}_{3 \min }$, however, were significantly greater than $\mathrm{CP}_{3.5 \%}(8,357 \pm 2,946 \mathrm{~J})$. The AWC values from the four protocols were highly inter-correlated at $0.76-0.91$.

\section{Conclusions}

The results of the present study indicated that $\mathrm{CP}$ and $\mathrm{AWC}$ could be estimated from a single, 3-min, all-out, test on a Monark cycle ergomter with the resistance set at 
$4.5 \%$ of the subject's body weight. In the present study, it was found that: 1) Unlike Vanhatalo et al. (44), there was a significant mean difference between estimates of CP from the $\mathrm{CP}_{\mathrm{PT}}$ and $\mathrm{CP}_{3 \min }$ tests, possibly due to the lower pedaling cadence selected in this study; and 2) CP and AWC could be accurately estimated from the $\mathrm{CP}_{4.5 \%}$ test, but not the $\mathrm{CP}_{3.5 \%}$ test. Thus, the advantages of the $\mathrm{CP}_{4.5 \%}$ test over the $\mathrm{CP}_{\mathrm{PT}}$ and $\mathrm{CP}_{3 \min }$ tests are: 1) The $\mathrm{CP}_{4.5 \%}$ tests requires only one workbout compared to multiple, exhaustive workbouts for the original $\mathrm{CP}$ test $\left(\mathrm{CP}_{\mathrm{PT}}\right)$ of Moritani et al. (35); 2) The $\mathrm{CP}_{4.5 \%}$ test utilizes a resistance set according to the body weight of the subject as opposed to the need to collect and analyze gas exchange variables during an incremental test to exhaustion to set the resistance for the $\mathrm{CP}_{3 \min }$ test of Burnley et al (10); and 3) The $\mathrm{CP}_{4.5 \%}$ test involves a single, 3-min, all-out, workbout on a Monark cycle ergometer, while the $\mathrm{CP}_{3 \min }$ test requires an electronically braked cycle ergometer. Therefore, the $\mathrm{CP}_{45 \%}$ test is recommended as a new practical and accurate method for estimating $\mathrm{CP}$ and AWC. 
Table 1. Descriptive Characteristics of the Subjects

\begin{tabular}{cccc}
\hline & weight $\mathbf{( k g )}$ & age (yrs) & Height $(\mathbf{c m})$ \\
\hline Females $(\mathrm{n}=6)$ & & & \\
1 & 62 & 20 & 168 \\
5 & 59 & 22 & 165 \\
7 & 56 & 23 & 173 \\
9 & 70 & 21 & 170 \\
10 & 55 & 21 & 163 \\
11 & 64 & 20 & 170 \\
Mean \pm SD & $\mathbf{6 1} \pm \mathbf{6}$ & $\mathbf{2 1} \pm \mathbf{1}$ & $\mathbf{1 6 8} \pm \mathbf{4}$ \\
Range & $\mathbf{5 5}-\mathbf{7 0}$ & $\mathbf{2 0}-\mathbf{2 3}$ & $\mathbf{1 6 3 - \mathbf { 1 7 3 }}$ \\
Males $(\mathrm{n}=6)$ & & & 178 \\
2 & 81 & 25 & 185 \\
3 & 70 & 21 & 180 \\
4 & 72 & 22 & 185 \\
6 & 86 & 23 & 188 \\
8 & 86 & 29 & 177 \\
12 & 81 & 31 & $\mathbf{1 8 2} \pm \mathbf{4}$ \\
Mean \pm SD & $\mathbf{7 9} \pm \mathbf{7}$ & $\mathbf{2 5} \pm \mathbf{4}$ & $\mathbf{1 7 7}-\mathbf{1 8 8}$ \\
\hline
\end{tabular}


Table 2. Mean $\dot{V} O_{2}$ max and peak power

\begin{tabular}{|c|c|c|c|}
\hline Subject & $\mathrm{VO}_{2} \mathrm{~L} \cdot \mathrm{min}^{-1}$ & $\mathrm{VO}_{2} \mathrm{~mL} \cdot \mathrm{kg} \cdot \mathrm{min}^{-1}$ & Peak Power (W) \\
\hline 1 & 2.67 & 43.06 & 210 \\
\hline 2 & 2.52 & 31.11 & 210 \\
\hline 3 & 3.18 & 45.20 & 140 \\
\hline 4 & 2.85 & 39.58 & 210 \\
\hline 5 & 2.15 & 36.44 & 180 \\
\hline 6 & 3.41 & 39.65 & 240 \\
\hline 7 & 1.74 & 31.07 & 150 \\
\hline 8 & 4.23 & 49.18 & 310 \\
\hline 9 & 3.47 & 49.57 & 270 \\
\hline 10 & 2.86 & 52.57 & 210 \\
\hline 11 & 3.06 & 47.81 & 240 \\
\hline 12 & 4.08 & 50.37 & 330 \\
\hline Mean & 3.02 & 42.97 & 225 \\
\hline SD & 0.73 & 7.42 & 58 \\
\hline Range & $1.74-4.23$ & $31.07-52.57$ & $180-330$ \\
\hline
\end{tabular}


Table 3. Female incremental cycling $\dot{V} O_{2}$ max values

\begin{tabular}{ccc}
\hline Subject & $\begin{array}{c}\mathbf{V O}_{\mathbf{2}} \\
\mathbf{L} \cdot \mathbf{m i n}^{-\mathbf{1}}\end{array}$ & $\begin{array}{c}\mathbf{V O} \\
\mathbf{m L} \cdot \mathbf{k g} \cdot \mathbf{m i n}^{-1}\end{array}$ \\
1 & 2.67 & 43.06 \\
5 & 2.15 & 36.44 \\
7 & 1.74 & 31.07 \\
9 & 3.47 & 49.57 \\
10 & 2.86 & 52.57 \\
11 & 3.06 & 47.81 \\
Mean & $\mathbf{2 . 6 6}$ & $\mathbf{4 3 . 4 2}$ \\
SD & $\mathbf{0 . 6 3}$ & $\mathbf{8 . 2 7}$ \\
\hline
\end{tabular}


Table 4. Male incremental cycling $\dot{V} O_{2}$ max values

\begin{tabular}{ccc}
\hline Suject & $\begin{array}{c}\mathbf{V O}_{\mathbf{2}} \\
\mathbf{L} \cdot \mathbf{m i n}^{-\mathbf{1}}\end{array}$ & $\begin{array}{c}\mathbf{V O} \\
\mathbf{m L} \cdot \mathbf{k g} \cdot \mathbf{m i n}^{-1}\end{array}$ \\
2 & 2.52 & 31.11 \\
3 & 3.18 & 45.20 \\
4 & 2.85 & 39.58 \\
6 & 3.41 & 39.65 \\
8 & 4.23 & 49.18 \\
12 & 4.08 & 50.37 \\
Mean & $\mathbf{3 . 3 8}$ & $\mathbf{4 2 . 5 2}$ \\
SD & $\mathbf{0 . 6 7}$ & $\mathbf{7 . 2 1}$ \\
\hline
\end{tabular}


Table 5. Gas Exchange Threshold

\begin{tabular}{|c|c|c|c|}
\hline & L-min-1 & mL·kg·min-1 & \%V02 max \\
\hline \multicolumn{4}{|l|}{ Subject } \\
\hline 1 & 1.91 & 30.80 & 72 \\
\hline 2 & 1.92 & 23.70 & 76 \\
\hline 3 & 2.33 & 33.29 & 74 \\
\hline 4 & 2.31 & 32.08 & 81 \\
\hline 5 & 1.51 & 25.59 & 70 \\
\hline 6 & 2.57 & 29.88 & 75 \\
\hline 7 & 1.43 & 25.54 & 82 \\
\hline 8 & 3.1 & 36.05 & 73 \\
\hline 9 & 2.09 & 29.86 & 60 \\
\hline 10 & 1.94 & 35.27 & 67 \\
\hline 11 & 2.2 & 34.38 & 72 \\
\hline 12 & 2.62 & 32.35 & 64 \\
\hline mean & 2.16 & 30.73 & 72 \\
\hline sd & 0.47 & 4.02 & 6 \\
\hline range & $1.51-3.10$ & 23.70-36.05 & 60-82 \\
\hline
\end{tabular}


Table 6. $\mathrm{W}_{\text {lim }}$ vs. $\mathrm{T}_{\text {lim }}$ Relationship

\begin{tabular}{cc}
\hline Subject & $\mathbf{r}^{\mathbf{2}}$ \\
\hline 1 & 0.9989 \\
2 & 0.9997 \\
3 & 0.9804 \\
4 & 0.9985 \\
5 & 0.9998 \\
6 & 0.9931 \\
7 & 0.9987 \\
8 & 0.9996 \\
9 & 0.9961 \\
10 & 0.9958 \\
11 & 0.9971 \\
12 & 0.9999 \\
Mean & $\mathbf{0 . 9 9 6 5}$ \\
SD & $\mathbf{0 . 0 0 5 5}$ \\
Range & $\mathbf{0 . 9 8 0 4 - 0 . 9 9 9 9}$ \\
\hline
\end{tabular}


Table 7. CP (W) and AWC (J) values from all four protocols

\begin{tabular}{ccccc}
\hline Test & \multicolumn{1}{c}{ CP } & \multicolumn{2}{c}{ AWC } & Range \\
Protocol & Mean \pm SD & Range & Mean \pm SD & Rang \\
$\mathrm{CP}_{\mathrm{PT}}$ & $178 \pm 47$ & $103-256$ & $13412 \pm 6247$ & $7596-25775$ \\
$\mathrm{CP}_{3 \min }$ & $193 \pm 54^{\mathrm{a}}$ & $101-301$ & $10895 \pm 2923$ & $7356-15265$ \\
$\mathrm{CP}_{3.5 \%}$ & $173 \pm 40$ & $104-243$ & $8357 \pm 2946^{\mathrm{b}}$ & $5083-12942$ \\
$\mathrm{CP}_{4.5 \%}$ & $186 \pm 44$ & $111-273$ & $9842 \pm 4394$ & $4250-17231$ \\
\hline
\end{tabular}

${ }^{a}$ significantly different from $\mathrm{CP}_{\mathrm{PT}}$ and $\mathrm{CP}_{3.5 \%}$

${ }^{\mathrm{b}}$ significantly different from $\mathrm{CP}_{\mathrm{PT}}$ and $\mathrm{CP}_{3 \min }$ 
Table 8. Correlation Matrix for CP (W)

\begin{tabular}{crrrrr}
\hline & \multicolumn{1}{c}{$C P_{P T}$} & \multicolumn{1}{c}{$C P_{3 \min }$} & \multicolumn{1}{c}{$C P_{3.5 \%}$} & \multicolumn{1}{c}{$P_{4.5 \%}$} \\
\hline $\mathrm{CP}_{\text {PT }}$ & 1.00 & & & & \\
$\mathrm{CP}_{3 \min }$ & 0.97 & 1.00 & & & \\
$\mathrm{CP}_{3.5 \%}$ & 0.95 & 0.96 & 1.00 & \\
$\mathrm{CP}_{4.5 \%}$ & 0.90 & 0.95 & 0.89 & 1.00 \\
\hline
\end{tabular}


Table 9. Correlation Matrix for AWC (J)

\begin{tabular}{|c|c|c|c|c|}
\hline & $C P_{P T}$ & $C P_{3 \min }$ & $C P_{3.5 \%}$ & $C P_{4.5 \%}$ \\
\hline $\mathrm{CP}_{\mathrm{PT}}$ & 1.00 & & & \\
\hline $\mathrm{CP}_{3 \min }$ & 0.91 & 1.00 & & \\
\hline $\mathrm{CP}_{3.5 \%}$ & 0.82 & 0.87 & 1.00 & \\
\hline $\mathrm{CP}_{4.5 \%}$ & 0.76 & 0.78 & 0.95 & 1.00 \\
\hline
\end{tabular}


Figure 1 a

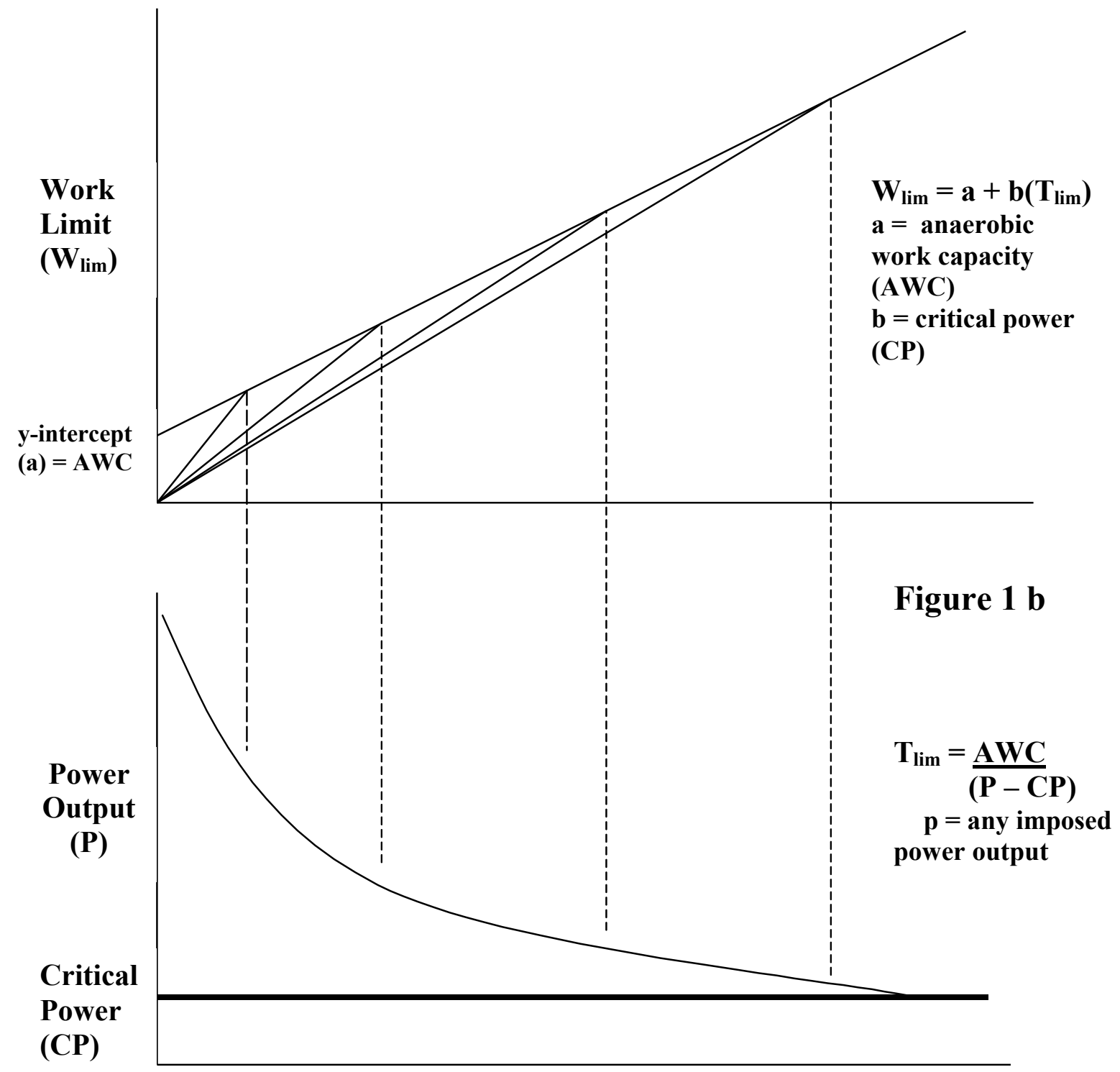

Time Iimit ( $\left.\mathrm{T}_{\mathrm{lim}}\right)$

Figure $1 \mathrm{a}$ and $1 \mathrm{~b}$. The relationship between work limit $\left(\mathrm{W}_{\text {lim }}\right)$ and time limit $\left(\mathrm{T}_{\text {lim }}\right)$ is described by the linear equation $\mathrm{W}_{\lim }=\mathrm{a}+\mathrm{b}\left(\mathrm{T}_{\mathrm{lim}}\right)$, where (a) is equal to $\mathrm{AWC}$ and $(\mathrm{b})$ is critical power $(\mathrm{CP})$. The equation $\mathrm{T}_{\mathrm{lim}}=\mathrm{AWC} /(\mathrm{P}-\mathrm{CP})$ describes the relationship between imposed power output $(\mathrm{P})$ versus $\mathrm{T}_{\text {lim }}$ for cycle ergometry and is derived from the linear relationship so that the asymptote is equivalent to ( $b$ or $\mathrm{CP}$ ). Theoretically, exercise can be maintained indefinitely when the imposed power output is $\leq \mathrm{CP}$. Time to exhaustion can be predicted for any power output $\geq \mathrm{CP}$ from the hyperbolic relationship, $\mathrm{T}_{\mathrm{lim}}=\mathrm{AWC} /(\mathrm{P}-\mathrm{CP})$. 


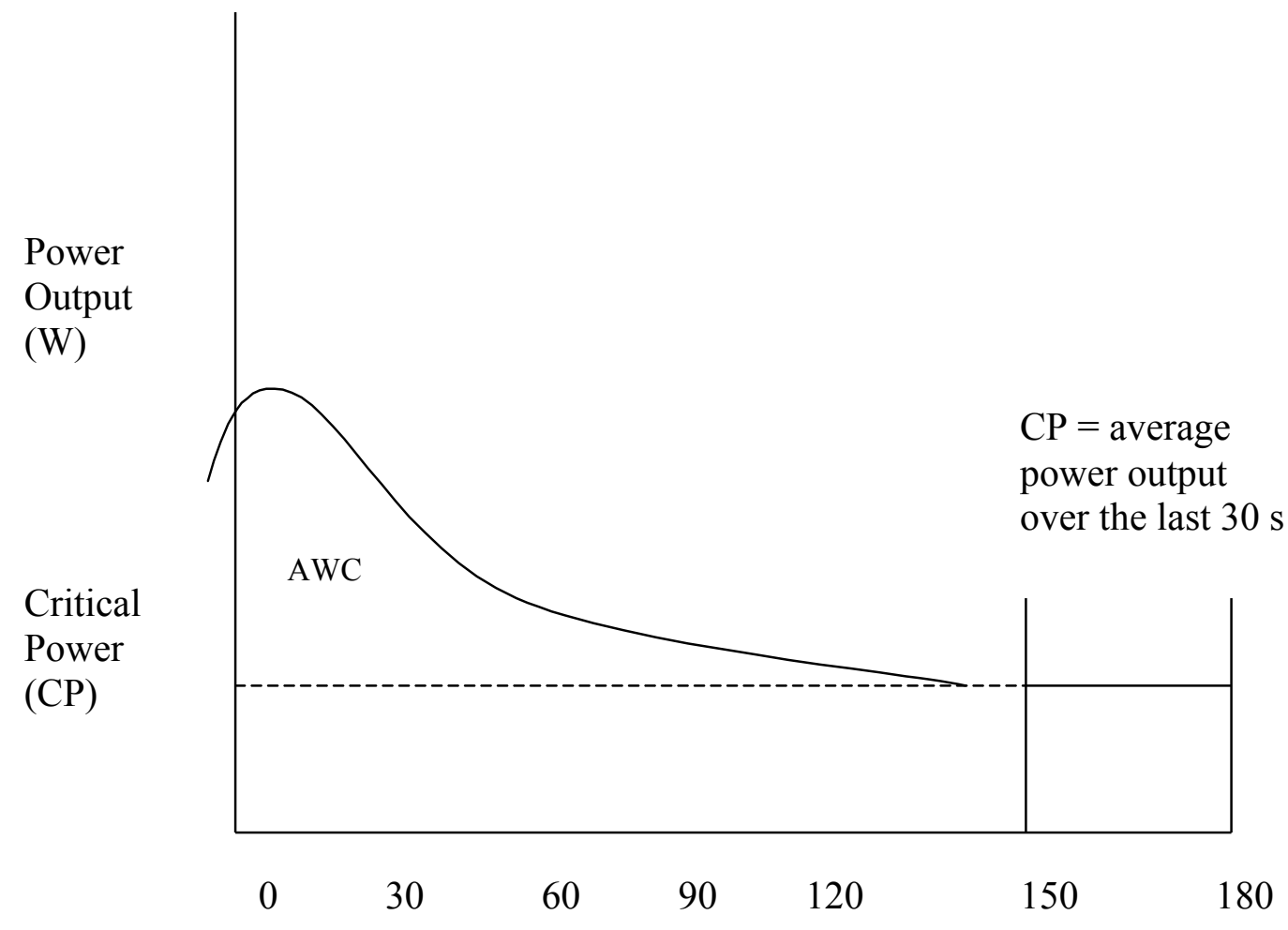

Time (s)

Figure 2. A schematic diagram of the power output (W) versus time (s) relationship for the 3-min all-out test. CP (W) is the average power output over the last $30 \mathrm{~s}$ $(150-180 \mathrm{~s})$ and AWC $(\mathrm{J})$ is the integral of the area under the curve above the CP. 


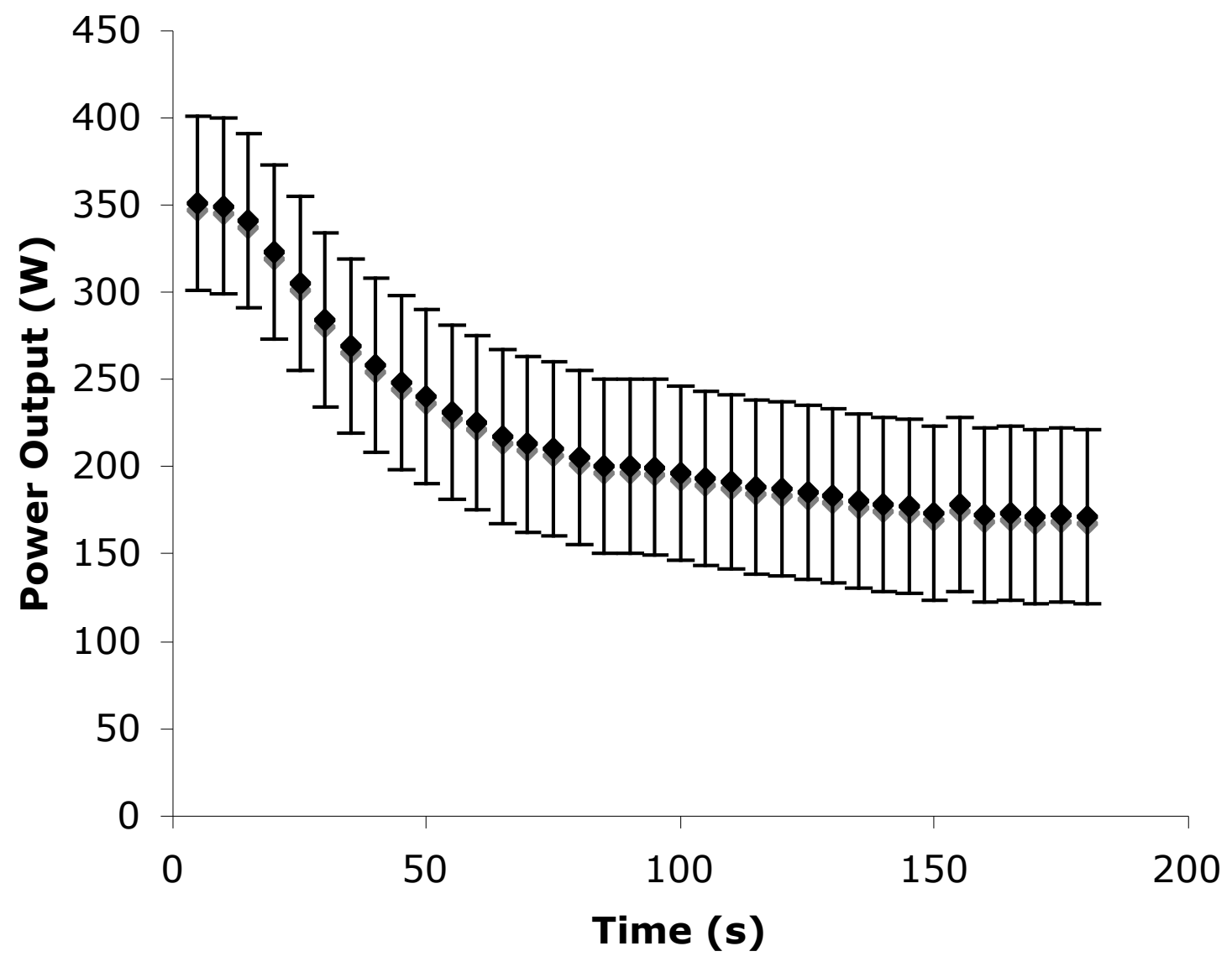

Figure 3. The mean ( $\pm \mathrm{SD}$ ) pattern of response for the $\mathrm{CP}_{3.5 \%}$.test 


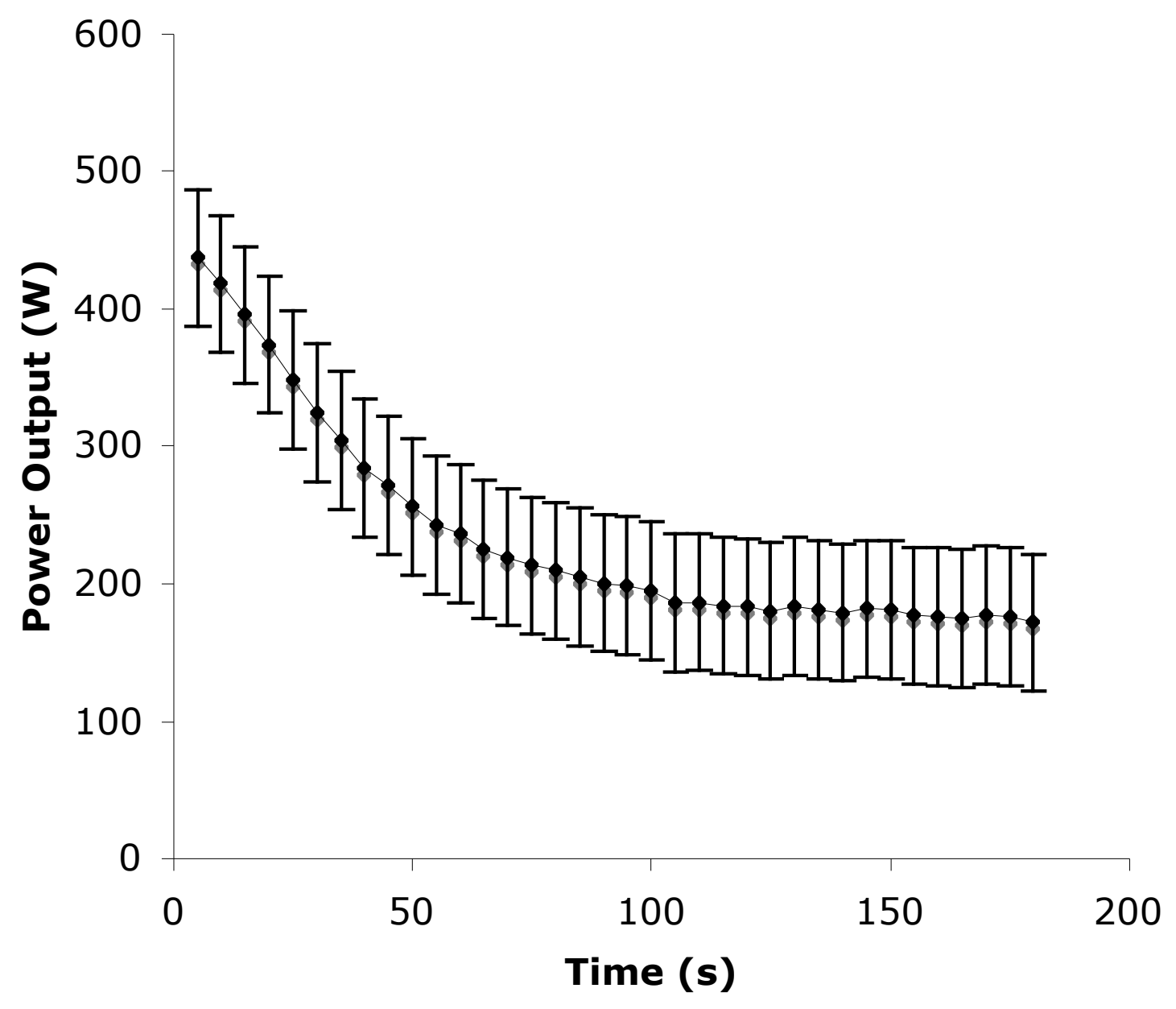

Figure 4. The mean ( $\pm \mathrm{SD}$ ) pattern of response for the $\mathrm{CP}_{4.5 \%}$.test 


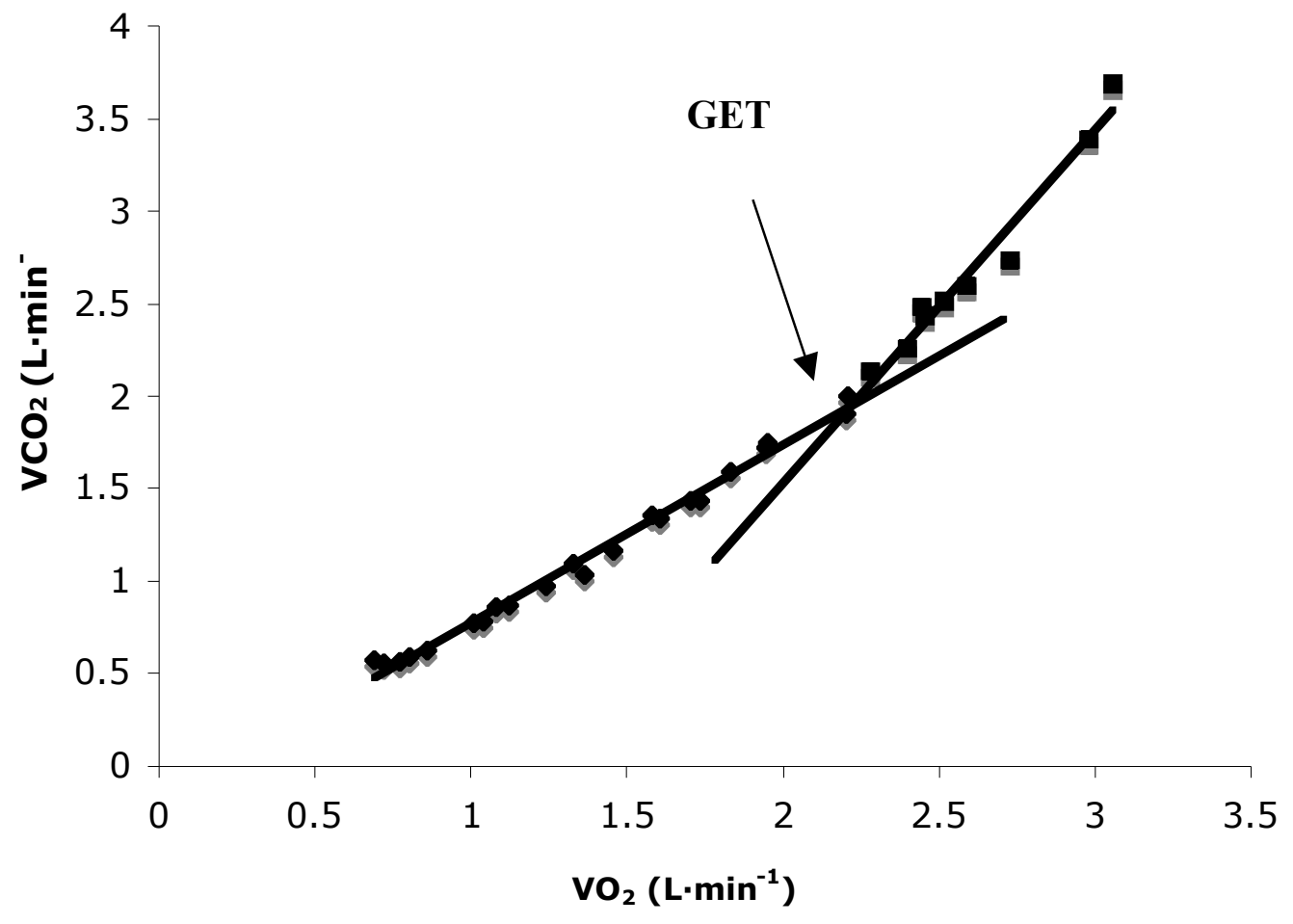

Figure 5. The method used for determining the GET 


\section{REFERENCES}

1. American College of Sports Medicine. Thompson, WR, Gordon, NF, and Pescatello, LS. ACSM's guidelines for exercise testing and prescription $8^{\text {th }}$ ed. Philadelphia, PA.: Lippincott Williams Wilkins, 2010, pp. 84-87.

2.Barker, T., Poole, DC, Noble, LM, and Barstow, TJ. Human critical power-oxygen uptake relationship at different pedaling frequencies. Exp Physiol 91: 621-632, 2006.

3. Beaver, WL, Wasserman, $\mathrm{K}$ and Whipp, BJ. A new method for detecting anaerobic threshold by gas exchange. J Appl Physiol 60:2020-2027, 1986.

4. Bishop, D, Jenkins, DG, Howard, A. The critical power function is dependent on the duration of the predictive exercise tests chosen. Int J Sports Med 19:125-129, 1998.

5. Brickley, G., Doust, J, and Williams, CA. Physiological responses during exercise to exhaustion at critical power. Eur J Appl Physiol 88:146-151, 2002.

6. Brickley, G, Dekerle, J, Hammond, AJ, Pringle, J, and Carter H. Assessment of maximal aerobic power and critical power in a single 90 -s isokinetic all-out cycling test. Int J Sports Med 28: 414-419, 2006.

7.Bulbulian, R, Wilcox, A, and Darabos, B. Anaerobic contributions to distance running performance of trained cross-country athletes. Med Sci Sports Excerc 18: 107-113, 1986.

8. Bulbulian, R, Jeong, JW, and Murphy, M. Comparison of aneorbic components of the Wingate and critical power tests in males and females. Med Sci Sports Exerc 28:1336-1341, 1996.

9. Bull, AJ, Housh, TJ, Johnson, GO, and Perry, SR. Effect of mathematical modeling on the estimation of critical power. Med Sci Sports Exerc 32: 526-530, 2000.

10. Burnley, M, Doust, JH, and Vanhatalo A. A 3-min all-out test to determine peak oxygen uptake and the maximal steady state. Med Sci Spots Exerc 38: 1995-2003, 2006.

11. Camic, CL, Housh, TJ, Meilke, M, Zuniga, JM, Hendrix, RC, Johnson, GO, Schmidt, RJ, and Housh, DJ. The effects of 4 weeks of an arginine-based supplement on the gas exchange threshold and peak oxygen uptake. Appl Physiol Nutr Metab 35: 286-293, 2010.

12. Carnvale, TJ, and Gaesser, GA. Effects of pedaling speed on the power-duration 
relationship for high-intensity exercise. Med Sci Sports Exerc 23: 242-246, 1991.

13. Davis, JA, Storer, TW, and Caiozo, VJ. Prediction of normal values for lactate threshold estimated by gas exchange in men and women. Eur J Appl Physiol 76:157 - 164, 1997.

14. Day, JR, Rossiter, HB, Coats, EM, Skasick, A, and Whipp, BJ. The maximally attainable $\dot{V} \mathrm{O}_{2}$ during exercise in humans: the peak vs. maximum issue. $J \mathrm{Appl}$ Physiol 95:1901-1907, 2003.

15. Gaesser, GA and Wilson, LA. Effects of continuous and interval training on the parameters of the power-endurance time relationship for high intensity exercise. Int J Sports Med 9:417-421, 1988.

16. Gaesser, GA, Carnevale, TJ, Garfinkel, A, Walter, DO, and Womack, CJ. Estimation of critical power with nonlinear and linear models. Med Sci Sports Excerc 27: 1430-1438, 1995.

17. Dekerle, J, Brickley, G, Hammond, AJP, Pringle, JSM, and Carter, H. Validity of the two-parameter model in estimating the anaerobic work capacity. Eur J Appl Physiol 96:257-264, 2006.

18. Fukuba, Y, Miura, A, Endo, M, Kan, A, Yanagawa, K, and Whipp, BJ. The curvature constant parameter of the power-duration curve for varied-power exercise. Med Sci Sports Exerc 35: 1413-1418, 2003.

19. Hill, WH and Smith, JC. A comparison of methods of estimating anaerobic work capacity. Ergonomics 36: 1495-1500, 1993.

20. Hill, WH and Smith, JC. Determination of critical power by pulmonary gas exchange. Can. J. Appl. Physiol 24: 74-86, 1999.

21. Hill, DW, Poole, PC, and Smith, JC. The relationship between power and the time to achieve $\dot{V} O_{2}$ max. Med Sci Sports Exerc 34: 709-714, 2002.

22. Housh, DJ, Housh, TJ, and Bauge, SM. The accuracy of the critical power test for predicting time to exhaustion during cycle ergometry. Ergonomics 32: 997-1004, 1989.

23. Housh, DJ, Housh, T, and Bauge, SM. A methodological consideration for the determination of critical power and anaerobic work capacity. Res $Q$ Exercise Sport 61: 406-409, 1990.

24. Housh, TJ, deVries, HA, Housh, DJ, Tichy, MW, Smyth, KD, and Tichy, AM. The relationship between critical power and the onset of blood lactate 
accumulation. J Sports Med Phys Fitness 31: 31-36, 1991.

25. Jenkins, DG and Quigley, BM. Blood lactate in trained cyclists during ergometry at critical power. Eur J Appl Physiol 61: 278-283, 1990.

26. Jenkins, DG and Quigley, BM. The y-intercept of the critical power function as a measure of anaerobic work capacity. Ergonomics 34:13-22, 1991.

27. Jenkins, DG and Quigley, BM. Endurance training enhances critical power. Med Sci Sports Exerc 24: 1283-1289, 1992.

28. Jenkins, DG and Quigley, BM. The influence of high intensity exercise training on the $\mathrm{W}_{\lim }-\mathrm{T}_{\lim }$ relationship. Med Sci Sports Exerc 25: 275-282, 1993.

29. Jones AM, Wilkerson, DP, DiMenna, F, Fulford, J and Poole, DC. Muscle metabolic responses to exercise above and below the "critical power" assessed using 31P-MRS. Am J Physiol Regul Integr Comp Physiol, 294: R585-93, 2008. Kelly, G.E. Estimation of the gas exchange threshold in humans: a time series approach. Eur J of Appl Physiol 85: 586 - 592, 2001.

30. Lucia, A, Hoyos, J, and Chicharro, JL. Preferred pedaling cadence in professional cycling. Med Sci Sports Exerc 33: 1361-1366, 2001.

31. Marsh, AP and Martin, PE. Effect of cycling experience, aerobic power, and power output on preferred and most economical cycling cadences. Med Sci Sports Exerc 29: 1225-1232, 1997.

32. Marsh, AP, Martin, PE, and Foley, KO. Effect of cadence, cycling experience, and aerobic power on delta efficiency during cycling. Med Sci Sports Exerc 32: 1630-1644, 2000.

33. McLellan, TM and Cheung, KSY. A comparative evaluation of the individual anaerobic threshold and the critical power. Med Sci Sports Exerc 24: 543-550. 1992.

34. Monod, H and Scherrer, J. The work capacity of a synergic muscular group. Ergonomics 8: 329-338, 1965.

35. Moritani, T, Nagata, A, deVries, HA and M. Muro. Critical power as a measure of physical work capacity and anaerobic threshold. Ergonomics 24: 339-350, 1981.

36. Nebelsick-Gullett, LJ, Housh, TJ, Johnson, GO, and Bauge, SM. A comparison between methods of measuring anaerobic work capacity. Ergonomics 31: 14131319, 1988. 
37. Poole, DC, Ward, AW, Gardner, GW, and Whipp, BJ. Metabolic and respiratory profile of the upper limit for prolonged exercise in man. Ergonomics 31: 1265 1279, 1988.

38. Poole, DC, Ward, SA, and Whipp, BJ. The effects of training on the metabolic and respiratory profile of high-intensity cycle ergometer exercise. Eur J Appl Physiol 59: 421-429, 1990.

39. Pringle, JSM and Jones, JM. Maximal lactate steady state, critical power, and EMG during cycling. Eur J Appl Physiol 88: 214-226, 2002.

40. Smith, JC and Hill, DW. Stability of parameter estimates derived from the power/time relationship. Can J App Physiol 18:43-47, 1991.

41. Smith, JC and Hill, DW. Mathematical models of the power-time relationship in high-intensity cycling. Abstract. Med Sci Sports Exerc 24: S74, 1992.

42. Solberg, G, Robstad, B, Sknonsberg, OH, and Borchsenius, F. Respiratory gas exchange indices for estimating the anaerobic threshold. J. Sports Sci. Med 4: 29$36,2005$.

43. Vandewalle, H, Kapitaniak, B, Grun, S, Raveneau, S, and Monod, H. Comparison between a 3-s all-out test and a time-work test on a cycle ergometer. Eur J Appl Physiology 58: 375-381, 1989.

44. Vanhatalo, A, Doust, DH, and Burnley, M. Determination of critical power using a 3-min all-out cycling test. Med Sci Sports Exerc 39: 548-555, 2007.

45. Vanhatalo, A, Doust, DH, and Burnley, M. A 3-min all-out cycling test is sensitive to a change in critical power. Med Sci Sports Exerc 40: 1693-1699, 2008 a.

46. Vanhatalo, A, Doust, DH, and Burnley, M. Robustness of a 3-min all-out cycling test to manipulations of power and cadence in humans. Exp Physiol 93: 383-390, 2008 b. 


\section{APPENDIX A}

\section{Glossary}

AWC anaerobic work capacity

CP critical power

$\mathrm{CP}_{\mathrm{PT}} \quad$ critical power test from the linear $\mathrm{W}_{\text {lim }}$ and $\mathrm{T}_{\text {lim }}$ relationship (35)

$\mathrm{CP}_{3 \min }$ three-minute all-out $\mathrm{CP}$ test on the electronically-braked cycle ergomter $(10,44)$

$\mathrm{CP}_{3.5 \%}$ three-minute all-out $\mathrm{CP}$ test on the Monark cycle ergometer with resistance set at $3.5 \%$ of the subject's body weight

$\mathrm{CP}_{4.5 \%}$ three-minute all-out $\mathrm{CP}$ test on the Monark cycle ergometer with resistance set at $4.5 \%$ of the subject's body weight

GET gas exchange threshold

J joules

P power output

$\mathrm{T}_{\lim } \quad$ time to exhaustion

W Watts

$\mathrm{W}_{\text {lim }} \quad$ work accomplished at exhaustion 


\section{APPENDIX B}

\section{Nebraskia Lincoln}

Human Performance Laboratory

Center for Youth Fitness and Sports Research

Department of Nutrition and Health Sciences

110 Ruth Leverton Hall

University of Nebraska-Lincoln

Lincoln, NE 68583-0806

Director: Terry J. Housh, Ph.D.

\section{Statement of Informed Consent}

\section{Title of Research Study}

A New Body Weight Protocol for the Estimation of Critical Power From a 3 Min All-Out Cycling Test.

\section{Invitation to Participate}

You are invited to participate in this research study. The following is provided in order to help you make an informed decision whether or not to participate. If you have any questions, please do not hesitate to ask.

\section{Basis for Subject Selection}

You were selected as a potential volunteer because you are between the ages of 19 and 29 years and in good health. If you wish to participate you must fill out a health history questionnaire. You will be prevented from participating in this research study if there are indications from the questionnaire that you may have health risks or if you are a pregnant female. Such indications include symptoms suggestive of chest pain, breathing difficulties, irregular heart beat, kidney or liver problems, high blood pressure or cholesterol, and/or abnormal electrocardiogram (EKG). Muscle or skeletal disorders including previous or current ankle, knee, and/or hip injuries may also preclude you from participation in this study. If you have no muscle/skeletal disorders or disease that will prevent you from engaging in physical activity, you will be asked to perform the tests described below. Overall, there are numerous health-related issues that may preclude you from participation in this study and inclusion will be determined on a subject-by-subject basis.

\section{Purpose of the Study}

The purpose of the present study is to develop a 3-minute test protocol for estimating critical power and anaerobic work capacity based on body weight using the Monark cycle ergometer. During the first session, you will be asked to perform a cycling test until voluntary exhaustion or you can no longer maintain a pedal cadence of $70 \mathrm{rev} \cdot \mathrm{min}^{-1}$. The maximal oxygen consumption ( $\mathrm{V}_{2} \max$ ), heart rate, and power output during a cycling test will be investigated. The following seven session will involve seven maximal cycling tests to exhaustion at varying power outputs. 


\section{Explanation of Procedures}

You will be asked to visit the Human Performance Laboratory located in Mabel Lee Hall (Room $141 \& 151$ ) on the UN-L campus on eight separate days, separated by $24-48$ hours. Visit 1, at the Body Composition Laboratory (151 Mabel Lee Hall), will consist of reading the informed consent, filling out a health history questionnaire, and deciding if you want to participate in the current study. You will then perform a cycling test on the electronically braked cycle ergometer (stationary bicycle) to determine peak oxygen consumption (VO2 max) and maximal heart rate (HRmax). During visits 2 through 5 (151 Mabel Lee Hall) you will perform one ride to exhaustion each visit at one of four, different, power outputs designed to elicit fatigue in a range of times from 1 to $15 \mathrm{~min}$. Visit 6 (151 Mabel Lee Hall) will consist of one 3-minute all out cycling test. During Visits 7 and 8 (151 Mabel Lee Hall) you will be asked to perform another 3-min all out cycling test on a different stationary bicycle (Monark cycle ergometer). To prevent pacing and ensure an all out effort, you will not be aware of the elapsed time and strong verbal encouragement will be provided.

\section{$\underline{\text { Total Time Commitment }}$}

The total time commitment for this study will be approximately 8.5 hours with each session lasting 1 hour to 1.5 hours.

\section{Potential Risks and Discomforts}

The following are the potential risks and discomforts you may experience during this study:

Cycling Tests - Pedaling on the exercise bike can cause aching in your legs, fatigue, sweating, shortness of breath and discomfort in the chest. However, you can stop cycling any time you want. Heavy exercise can cause high or low blood pressure, fainting, irregular heart rhythm, chest pain, and very rarely, heart attack, stroke or cardiac arrest. The need for hospital admission is reported in less than six of every 10,000 exercise tests. Cardiac arrest is reported in less than one of every 10,000 exercise tests.

\section{Protection Against Risks}

To minimize any potential risks and/or discomforts, you will be given instructions for special stretches, which may aid in the elimination of any muscle soreness as a result of the tests. In addition, you will be asked repeatedly during the tests how you feel in relation to your ability to continue the test. Throughout all the tests, you will be monitored by laboratory personnel trained in Cardiopulmonary Pulmonary Resuscitation (CPR) and use of an Automated External Defibrillator (AED). In addition, you will be asked repeatedly if you feel you can continue the tests.

\section{Potential Benefits to Subjects}

Your main benefit from participating in this study may be feedback on your level of physical fitness.

\section{Subject Compensation}


You will receive a $\$ 50$ stipend for the completion of the eight visits. Your social security number will be required for tax purposes.

\section{Potential Benefits to Society}

Although there are not direct benefits by participating in this study, society may benefit from this research by having a better understanding of how to conduct scientificallybased exercise programs in sport, rehabilitative, and recreational settings.

\section{In Case of Emergency Contact Procedures}

In the event of a research-related injury, immediately contact one of the investigators listed at the end of this consent form.

\section{Medical Care in Case of Injury}

In the unlikely event that you should suffer an injury as a direct consequence of the research procedures described above, the acute medical care required to treat the injury can be provided at the University of Nebraska Health Center from the hours of 8:00 a.m.-6:00 p.m. Monday through Friday, and 9:00 a.m.-12:30 p.m. Saturday (for urgent care needs only). The cost of such medical care will be the responsibility of the subject, whether at the University Health Center or at other local health care facilities. If the health center is unable to treat you, emergency care is available at local community health providers.

\section{Assurance of Confidentiality}

Any information obtained from this study which could identify you will be kept strictly confidential. The information may be published in scientific journals or presented at scientific meetings, but your identity will be kept strictly confidential. All data collected as a result of your participation will be kept in a locked cabinet in the office of the primary investigator (Room 141 Mabel Lee Hall). Your data will receive an identifying number and only the investigators will be able to identify you from your data. Your data will be compiled and only group data will be used for dissemination without identifying your name. For the purposes of future reference, your data will be stored for a minimum of 15 years. The social security information, however, will be kept separate from the rest of the data in a locked cabinet in the office of the primary investigator (Room $141 \mathrm{Mabel}$ Lee Hall) until you receive your payment, then this form will be shredded. 


\section{$\underline{\text { Rights of Research Subjects }}$}

You may ask any questions concerning this research and have those questions answered before agreeing to participate in or during the study. Or you may call the investigator, Haley Bergstrom, at any time, office phone, (402) 472-2690, or after hours (308) 3251363. You may also contact Dr. Terry Housh at his office phone, (402) 472-1160, or after hours (402) 477-6573. Please contact the investigator:

- if you want to voice concerns or complaints about the research

- in the event of a research related injury.

Please contact the University of Nebraska-Lincoln Institutional Review Board at (402) 472-6965 for the following reasons:

- you wish to talk to someone other than the research staff to obtain answers to questions about your rights as a research participant

- to voice concerns or complaints about the research

- to provide input concerning the research process

- in the event the study staff could not be reached.

\section{Voluntary Participation Withdrawal}

You are free to decide not to participate in this study, or to withdraw at any time without adversely affecting your relationship with the investigators or the University of Nebraska. Your decision will not result in any loss of benefits to which you are otherwise entitled 
You are voluntarily making a decision whether or not to participate in this research study. Your signature certifies that the content and meaning of the information on this consent form have been fully explained to you and that you have decided to participate having read and understood the information presented. Your signature also certifies that you have had all your questions answered to your satisfaction. If you think of any questions during this study, please contact the investigators. You will be given a copy of this consent form to keep.

Signature of Research Participant

Date

Printed name of Research Participant

My signature as witness certifies that the subject signed this consent form in my presence as his/her voluntary act and deed.

Signature of Investigator

Date

Investigators:

Haley Bergstrom

work phone

(402) 472-2690

home phone (308) 325-1363

Terry Housh

work phone

(402) $472-1160$

home phone (402) 477-6573

Glen Johnson

work phone (402) 472-2690

home phone (402) 423-6443 


\section{PRE-EXERCISE TESTING HEALTH STATUS QUESTIONNAIRE}

Name

Date

Home Address

Work Phone

Home Phone

E-mail address

Person to contact in case of emergency

Emergency Contact Phone

Personal Physician

Gender

Age

(yrs) Height

Birthday (mm/dd/yy)

Physician's Phone

Does the above weight indicate: a gain year?

If a change, how many pounds?

(in) Weight

(lbs)

A. JOINT-MUSCLE STATUS ( $\checkmark$ Check areas where you currently have problems)

$\underline{\text { Joint Areas }}$

( ) Wrists

( ) Elbows

( ) Shoulders

( ) Upper Spine \& Neck

( ) Lower Spine

( ) Hips

( ) Knees

( ) Ankles

( ) Feet

( ) Other a loss

no change in the past (lbs)
Muscle Areas

( ) Arms

( ) Shoulders

( ) Chest

( ) Upper Back \& Neck

( ) Abdominal Regions

( ) Lower Back

( ) Buttocks

( ) Thighs

( ) Lower Leg

( ) Feet

( ) Other

B. HEALTH STATUS ( $\checkmark$ Check if you previously had or currently have any of the following conditions)

( ) High Blood Pressure

( ) Heart Disease or Dysfunction

( ) Peripheral Circulatory Disorder

( ) Lung Disease or Dysfunction

( ) Arthritis or Gout

( ) Edema

( ) Epilepsy

( ) Multiply Sclerosis

( ) High Blood Cholesterol or Triglyceride Levels
( ) Acute Infection

( ) Diabetes or Blood Sugar Level Abnormality

( ) Anemia

( ) Hernias

( ) Thyroid Dysfunction

( ) Pancreas Dysfunction

( ) Liver Dysfunction

( ) Kidney Dysfunction

( ) Phenylketonuria (PKU)

( ) Allergic Reactions to Medication

( ) Loss of Consciousness please describe

( ) Others That You Feel We Should Know About

( ) Pregnant

( ) Allergic Reactions to Any Other Substance please describe 
C. PHYSICAL EXAMINATION HISTORY

Approximate date of your last physical examination

Physical problems noted at that time

Has a physician ever made any recommendations relative to limiting your level of physical exertion? YES NO

If YES, what limitations were recommended?

Have you ever had an abnormal resting electrocardiogram (ECG)? YES $\mathrm{NO}$

D. CURRENT MEDICATION USAGE (List the drug name and the condition being managed)

MEDICATION

CONDITION

E. PHYSICAL PERCEPTIONS (Indicate any unusual sensations or perceptions. $\checkmark$ Check if you have recently experienced any of the following during or soon after physical activity (PA); or during sedentary periods (SED))

\begin{tabular}{|c|c|c|c|}
\hline PA & $\underline{\text { SED }}$ & $\underline{\text { PA }}$ & $\underline{\mathrm{SED}}$ \\
\hline () & ( ) Chest Pain & $(\mathrm{r})$ & ( ) Nausea \\
\hline ) & ( ) Heart Palpitations "fast irregular heart beats" & ( ) & ( ) Light Headedness \\
\hline ( ) & ( ) Unusually Rapid Breathing & ( ) & ( ) Loss of \\
\hline \multicolumn{4}{|c|}{ Consciousness } \\
\hline$($ ) & ( ) Overheating & ( ) & ( ) Loss of Balance \\
\hline ( ) & ( ) Muscle Cramping & ( ) & ( ) Loss of \\
\hline \multicolumn{4}{|c|}{ Coordination } \\
\hline Weakness & $\begin{array}{l}\text { ( ) Muscle Pain } \\
\text { ess }\end{array}$ & ( ) & ( ) Extreme \\
\hline() & ( ) Joint Pain & $($ ) & ( ) Numbness \\
\hline ( ) & ( ) Other & ( ) & ( ) Mental Confusion \\
\hline
\end{tabular}

F. FAMILY HISTORY $(\checkmark$ Check if any of your blood relatives . . p parents, brothers, sisters, aunts, uncles, and/or grandparents . . . have or had any of the following)

( ) Heart Disease

( ) Heart Attacks or Strokes (prior to age 50)

( ) Elevated Blood Cholesterol or Triglyceride Levels

( ) High Blood Pressure

( ) Diabetes

( ) Sudden Death (other than accidental) 
G. CURRENT HABITS ( $\checkmark$ Check any of the following if they are characteristic of you current habits)

( ) Smoking. If so, how many per day?

( ) Regularly does manual garden or yard work

( ) Regularly goes for long walks

( ) Frequently rides a bicycle

( ) Frequently runs/jogs for exercise

( ) Regularly participates in a weight training exercise program

( ) Engages in a sports program more than once per week.

If so, what does the program consist of? 